TITLE:

\title{
Geometric Gaussianity and non- Gaussianity in the cosmic microwave background
}

$\operatorname{AUTHOR}(S)$ :

Inoue, KT

CITATION:

Inoue, KT. Geometric Gaussianity and non-Gaussianity in the cosmic microwave background. PHYSICAL REVIEW D 2000, 62(10): 103001.

ISSUE DATE:

2000-11-15

URL:

http://hdl.handle.net/2433/50443

RIGHT:

Copyright 2000 American Physical Society 
PHYSICAL REVIEW D, VOLUME 62, 103001

\title{
Geometric Gaussianity and non-Gaussianity in the cosmic microwave background
}

\author{
Kaiki Taro Inoue \\ Yukawa Institute for Theoretical Physics, Kyoto University, Kyoto 606-8502, Japan
}

(Received 18 February 2000; published 3 October 2000)

\begin{abstract}
In this paper, the Gaussianity of eigenmodes and non-Gaussianity in the cosmic microwave background $(\mathrm{CMB})$ temperature fluctuations in the two smallest compact hyperbolic $(\mathrm{CH})$ models are investigated. First, it is numerically found that the expansion coefficients of low-lying eigenmodes on the two $\mathrm{CH}$ manifolds behave as if they are Gaussian random numbers in almost all places. Next, the non-Gaussianity of the temperature fluctuations in the $(l, m)$ space in these models is studied. Assuming that the initial fluctuations are Gaussian, the real expansion coefficients $b_{l m}$ of the temperature fluctuations in the sky are found to be distinctively non-Gaussian. In particular, the cosmic variances are found to be much larger than for Gaussian models. On the other hand, the anisotropic structure is vastly erased if one averages the fluctuations at a number of different observation points because of the Gaussian pseudorandomness of the eigenmodes. Thus the dominant contribution to the two-point correlation functions comes from the isotropic terms described by the angular power spectra $C_{l}$. Finally, topological quantities, the total length and the genus of isotemperature contours are investigated. The variances of total length and genus at high and low threshold levels are found to be considerably larger than that of Gaussian models while the means almost agree with them.
\end{abstract}

PACS number(s): 98.70.Vc, 98.80.Hw

\section{INTRODUCTION}

In recent years, locally isotropic and homogeneous Friedmann-Robertson-Walker (FRW) models with a nontrivial topology have attracted much attention. In the standard scenario, a simple connectivity of the spatial hypersurface is assumed for simplicity. However, the Einstein equations, being local equations, do not fix the global topology of the spacetime. In other words, a wide variety of topologically distinct spacetimes with the same local geometry described by a local metric element remain unspecified (see Ref. [1] for review on the cosmological topology). The determination of the global topology of the universe is one of the most important problem of the modern observational cosmology.

For flat models without the cosmological constant, severest constraints have been obtained by using the cosmic background explorer (COBE) differential microwave radiometer (DMR) data. The suppression of the fluctuations on scales beyond the topological identification scale $L$ leads to a decrease in the angular power spectra $C_{l}$ of the cosmic microwave background $(\mathrm{CMB})$ temperature fluctuations on large angular scales which puts a lower bound $L \geqslant 2400 h^{-1} \mathrm{Mpc}$ (with $h=H_{0} / 100 \mathrm{~km} \mathrm{~s}^{-1} \mathrm{Mpc}^{-1}$ ) for a compact flat threetorus model without a cosmological constant $[2,3]$. Similar constraints have been obtained for other compact flat models [4]. The maximum expected number of copies of the fundamental domain (cell) inside the last scattering surface is approximately 8 for the three-torus model.

In contrast, for low density models, the constraint could be considerably milder than the locally isotropic and homogeneous flat (Einstein-de Sitter) models since a bulk of large-angle CMB fluctuations can be produced by the socalled (late) integrated Sachs-Wolfe (ISW) effect $[5,6]$ which is the gravitational blueshift effect of the free streaming photons by the decay of the gravitational potential. As the gravitational potential decays in either $\Lambda$-dominant epoch or cur- vature dominant epoch, the free streaming photons with large wavelength (the light travel time across the wavelength is greater than or comparable to the decay time) that climbed a potential well at the last scattering experience blueshifts due to the contraction of the comoving space along the trajectories of the photons. Because the angular sizes of the fluctuations produced at late time are large, the suppression of the fluctuations on scale larger than the topological identification scale does not lead to a significant suppression of the largeangle power if the ISW effect is dominant. Recent works $[7-10]$ have shown that the large-angle powers $(2 \leqslant l \leqslant 20)$ are completely consistent with the COBE DMR data for compact hyperbolic $(\mathrm{CH})$ models which include a small $\mathrm{CH}$ orbifold and Weeks and the Thurston manifolds with volume $0.72,0.94$, and 0.98 in units of the cube of the curvature radius, respectively. Note that the Weeks manifold is the smallest and the Thurston manifolds is the second smallest in the known $\mathrm{CH}$ manifolds. For instance, the number of copies of the fundamental domain inside the last scattering surface at present is approximately 190 for a Weeks model with $\Omega_{0}=0.3$.

If the space is negatively curved, for a fixed number of the copies of the fundamental domain inside the present horizon, the large-angle fluctuations can be produced very effectively. In negatively curved spaces (hyperbolic spaces), trajectories of photons subtend a much smaller angle in the sky for a given scale. In other words, for a given angle of a pair of two photon trajectories, the physical distance of the trajectories is much greater than that in flat space. Therefore, even if there is a number of copies of the fundamental domain which intersect the last scattering surface, the number of copies which intersect the wave front (a sphere with $z=$ const) of the free streaming photons is exponentially decreased at late time when the large-angle fluctuations are produced due to the ISW effect.

However, one may not be satisfied with the constraints using only the angular power spectrum $C_{l}$ since it contains 
only isotropic information of the ensemble averaged temperature fluctuations [11]. If they have anisotropic structures, non-Gaussian signatures must be revealed. In fact, the global isotropy of the locally isotropic and homogeneous FRW models is generally broken. For instance, a flat three-torus obtained by identifying the opposite faces of a cube is obviously anisotropic at any points. Thus the temperature fluctuations averaged over the initial conditions in these multiply connected FRW models are no longer $\mathrm{SO}(3)$ invariant at a certain point. The temperature fluctuations on the sky are written in terms of (real) spherical harmonics $Q_{l m}(\mathbf{n})$ as

$$
\frac{\Delta T}{T}(\mathbf{n})=\sum_{l} \sum_{m=-l}^{l} b_{l m} Q_{l m}(\mathbf{n}) .
$$

If the distribution functions of the real expansion coefficients $b_{l m}$ are $\mathrm{SO}(3)$ invariant, the temperature fluctuations must be Gaussian provided that $b_{l m}$ 's are independent random numbers [12]. Therefore, the temperature fluctuations at a certain point in the multiply connected FRW models are not Gaussian if $b_{l m}$ 's are independent.

For the simplest flat three-torus models (without rotations in the identification maps) which are globally homogeneous, it is sufficient to choose one observing point and estimate how the power is distributed among the $m$ 's for a given angular scale $l$ in order to see the effect of the global anisotropy. However, in general, one must consider an ensemble of fluctuations at different observing points because of the spatial (global) inhomogeneity. In previous analyses, the dependence of the temperature fluctuations on the choice of observation points has not been fully investigated.

The lack of analytical results on the eigenmodes makes it difficult to investigate the nature of the temperature fluctuations in $\mathrm{CH}$ models. However, we may expect a high degree of complexity in the eigenmodes since the corresponding classical systems (geodesic flows) are strongly chaotic. In fact, it has been numerically found that the expansion coefficients of the low-lying eigenmodes on the Thurston manifold at the point where the injectivity radius is maximal are Gaussian pseudorandom numbers [13] which supports the previous analysis of the excited states (higher modes) of a two-dimensional asymmetrical $\mathrm{CH}$ model [14]. We have put a prefix "pseudo" since the eigenmodes are actually constrained by the periodic boundary conditions. These results imply that the statistical properties of the eigenmodes on $\mathrm{CH}$ spaces (orbifolds and manifolds) can be described by random-matrix theory (RMT) $[15,16]$. An investigation of the dependence of the property on the observation points is also important since $\mathrm{CH}$ spaces have symmetries (isometric groups) which may veil the random feature of the eigenmodes. In this paper, a detailed analysis on the statistical property of low-lying eigenmodes on the Weeks and the Thurston manifolds is conducted.

Assuming that the eigenmodes are Gaussian, one can expect that the anisotropic structure in the $(l, m)$ space is vastly erased when one averages the fluctuations over the space. This seems to be a paradox since the $\mathrm{CH}$ spaces are actually globally anisotropic. However, one should consider a spatial average of fluctuations with different initial conditions if one believes the Copernican principle that we are not in the center of the universe. Even if the space is anisotropic at a certain point, the averaged fluctuations may look isotropic by considering an ensemble of fluctuations at all the possible observing points. Note that the eigenmodes on $\mathrm{CH}$ spaces have no particular directions if they are Gaussian.

If the initial fluctuations are constant for each eigenmode, as we shall see, the Gaussian randomness of the temperature fluctuations can be solely attributed to the Gaussian pseudorandomness of the eigenmodes. In this case, the Gaussian randomness of the temperature fluctuations has its origin in the geometrical property of the space (geometric Gaussianity). Choosing an observing point is equivalent to fixing a certain initial condition. However, it is much natural to assume that the initial fluctuations are also random Gaussian as the standard inflationary scenarios predict. Then the temperature fluctuations may not obey the Gaussian statistics because they are written in terms of products of two different independent Gaussian numbers rather than sums while they remain almost spatially isotropic if averaged over the space.

In this paper, the Gaussianity of eigenmodes and nonGaussianity in the CMB for two smallest $\mathrm{CH}$ models (the Weeks and the Thurston models) are investigated. In Sec. II, numerical results on Gaussianity of eigenmodes are shown and we discuss to what extent the results are generic. In Sec. III, we study the non-Gaussian behavior of the temperature fluctuations in the $(l, m)$ space. In Sec. IV, topological quantities (total length and genus) of isotemperature contours are numerically simulated for studying the non-Gaussian behavior in the real space. Finally, we summarize our conclusions in Sec. V.

\section{GEOMETRIC GAUSSIANITY}

In locally isotropic and homogeneous FRW background spaces, each type (scalar, vector, and tensor) of first-order perturbations can be decomposed into a decoupled set of equations. In order to solve the decomposed linearly perturbed Einstein equations, it is useful to expand the perturbations in terms of eigenmodes of the Laplacian which satisfies the Helmholtz equation with certain boundary conditions

$$
\left(\nabla^{2}+k^{2}\right) u_{k}(x)=0
$$

since each eigenmode evolves independently in the linear approximation. Then one can easily see that the time evolution of the perturbations in the multiply connected locally isotropic and homogeneous FRW spaces coincide with that in the FRW spaces while the global structure of the background space is described solely by these eigenmodes.

Unfortunately, no analytical expressions of eigenmodes on $\mathrm{CH}$ spaces have been known. Nevertheless, the correspondence between classical and quantum mechanics may provide us a clue for understanding the generic property of the eigenmodes. If one recognizes the Laplacian as the Hamiltonian in a quantum system, each eigenmode can be interpreted as a wave function in a stationary state. Because classical dynamical systems (=geodesic flows) on $\mathrm{CH}$ spaces are strongly chaotic (or more precisely they are $K$ 
systems with ergodicity, mixing and Bernoulli properties [17]), one can expect a high degree of complexity for each eigenstate. The imprint of the chaos in the classical systems may be hidden in the quantum counterparts. In fact, in many cases, the short-range correlations observed in the eigenvalues (energy states) have been found to be consistent with the universal prediction of RMT for three universality classes: the Gaussian orthogonal ensemble (GOE), the Gaussian unitary ensemble (GUE), and the Gaussian symplectic ensemble (GSE) $[15,16]$. In our case the statistical properties are described by GOE (which consist of real symmetric $N \times N$ matrices $H$ which obey the Gaussian distribution $\propto \exp \left[-\operatorname{Tr} H^{2} /\left(4 a^{2}\right)\right]$ (where $a$ is a constant) as the systems possess a time-reversal symmetry. RMT also predicts that the squared expansion coefficients of an eigenstate with respect to a generic basis are distributed as Gaussian random numbers [18]. Unfortunately, no analytic forms of generic bases (=eigenmodes) are known for $\mathrm{CH}$ spaces which seems to be an intractable problem. However, if the eigenmodes are continued onto the universal covering space by the periodic boundary conditions, they can be written in terms of a "generic" basis on the universal covering space (=threehyperboloid $\left.H^{3}\right)$. In pseudospherical coordinates $(R, \chi, \theta, \phi)$, the eigenmodes are written in terms of complex expansion coefficients $\xi_{\nu l m}$ and eigenmodes on the universal covering space,

$$
u_{\nu}=\sum_{l m} \xi_{\nu l m} X_{\nu l}(\chi) Y_{l m}(\theta, \phi),
$$

where $\nu=\sqrt{k^{2}-1}, X_{\nu l}$, and $Y_{l m}$ denote the radial eigenfunction and (complex) spherical harmonic on the pseudosphere with radius $R$, respectively. Then the real expansion coefficients $a_{\nu l m}$ are given by

$$
\begin{aligned}
& a_{\nu 00}=-\operatorname{Im}\left(\xi_{\nu 00}\right), \quad a_{\nu l 0}=\sqrt{c_{\nu l}} \operatorname{Re}\left(\xi_{\nu l 0}\right), \\
& a_{\nu l m}=\sqrt{2} \operatorname{Re}\left(\xi_{\nu l m}\right), \quad m>0, \\
& a_{\nu l m}=-\sqrt{2} \operatorname{Im}\left(\xi_{\nu l-m}\right), \quad m<0,
\end{aligned}
$$

where

$$
\begin{aligned}
c_{\nu l} & =\frac{2}{\{1+\operatorname{Re}[F(\nu, l)]\}}, \\
F(\nu, l) & =\frac{\Gamma(l+\nu i+1)}{\Gamma(\nu i)} \frac{\Gamma(-\nu i)}{\Gamma(l-\nu i+1)} .
\end{aligned}
$$

In this paper, the low-lying eigenmodes $(k<13)$ on the Weeks and Thurston manifolds are numerically computed by the direct boundary element method. The identification matrices of the Dirichlet domains are obtained by a computer program "'SNAPPEA" by Weeks [19]. The computed eigenvalues are well consistent with that in the previous literature $[13,20]$. The estimated errors in $k$ are within 0.01 . However, the last digits in $k$ may be incorrect. $a_{\nu l m}$ 's can be promptly obtained after the normalization and orthogonalization of these eigenmodes. The orthogonalization is achieved at the

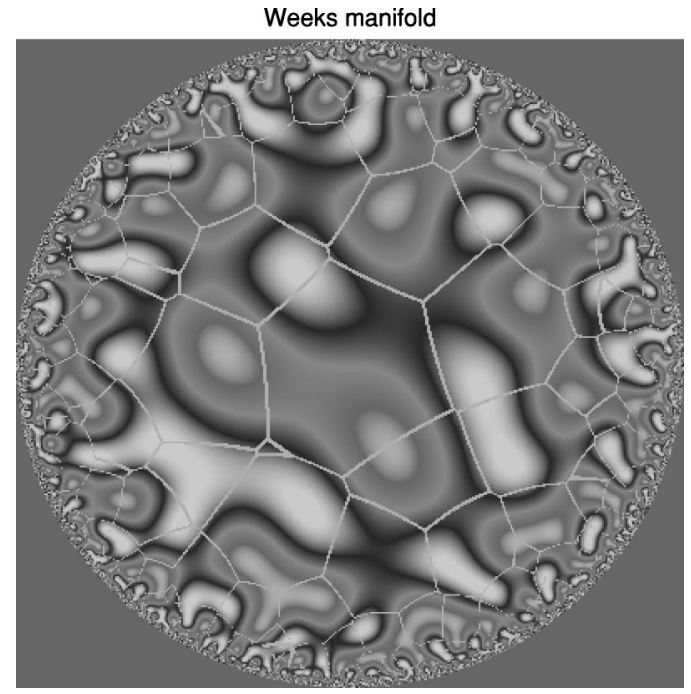

FIG. 1. The lowest eigenmode $k=5.268$ on the Weeks manifold continued onto the Poincaré ball and the boundaries of the copied Dirichlet domains (solid curves) plotted on a slice $z=0$.

level of $10^{-3}$ to $10^{-4}$ (for the inner product of the normalized eigenmodes) which implies that each eigenmode is computed with relatively high accuracy. In Figs. 1 and 2, one can see a high degree of complexity in the lowest eigenmodes on the Poincare ball which is isometric given by

$$
\begin{aligned}
& x=R \tanh \frac{\chi}{2} \sin \theta \cos \phi, \quad y=R \tanh \frac{\chi}{2} \sin \theta \sin \phi, \\
& z=R \tanh \frac{\chi}{2} \cos \theta .
\end{aligned}
$$

Replacing $\tanh (\chi / 2)$ by $\tanh \chi$ for each coordinate, one obtains the Klein (projective) coordinates. In the Poincar e coordinates, angles of geodesics coincide with that of Euclid-

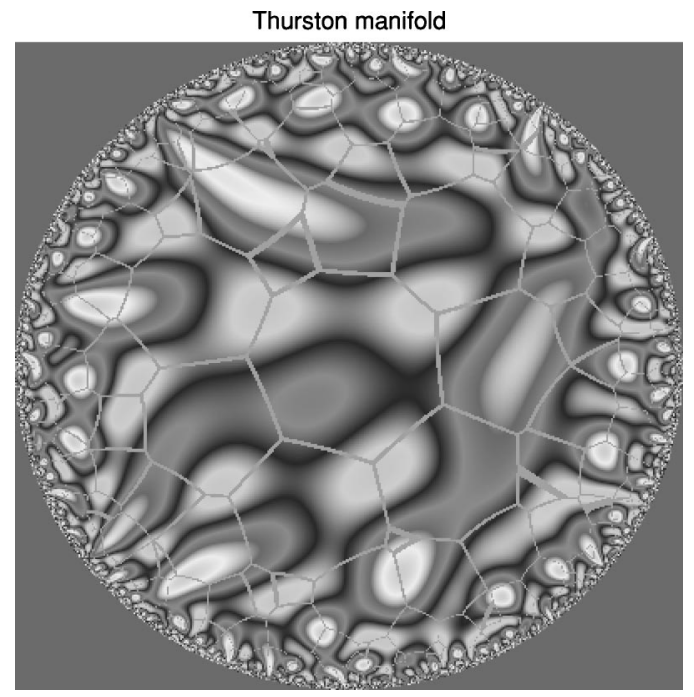

FIG. 2. The lowest eigenmode $k=5.404$ on the Thurston manifold continued onto the Poincare ball and the boundaries of the copied Dirichlet domains (solid curves) plotted on a slice $z=0$. 

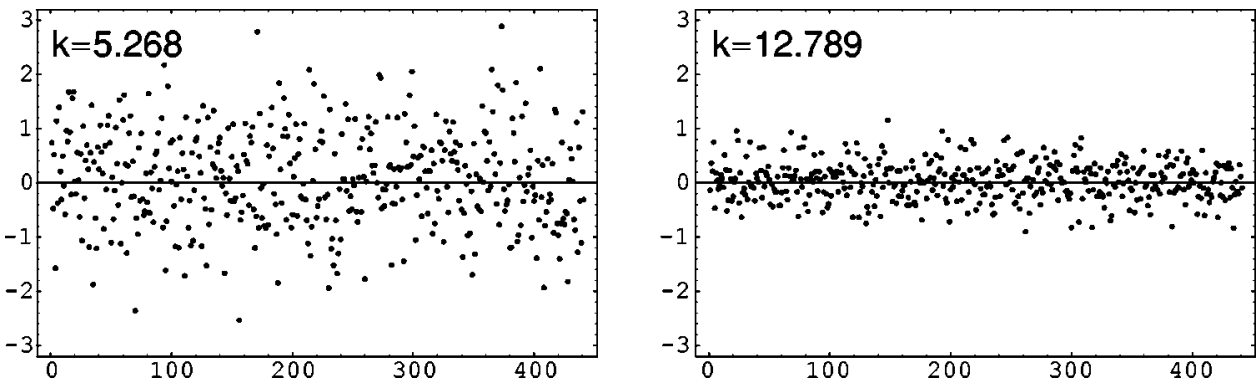

FIG. 3. Plots of $a_{\nu l m}$ 's which are ordered as $l(l+1)+m+1,0$ $\leqslant l \leqslant 20$ for eigenmodes $k=5.268$ (left) and $k=12.789$ (right) on the Weeks manifold at a point which is randomly chosen. ean ones. In the Klein coordinates, all geodesics are straight lines while angles does not coincide with that of Euclidean ones. In what follows $R$ is normalized to 1 without loss of generality.

In Fig. 3, one can see that the distribution of $a_{\nu l m}$ 's which are ordered as $l(l+1)+m+1$ are qualitatively random. In order to estimate the randomness quantitatively, we consider a cumulative distribution of

$$
b_{\nu l m}=\frac{\left|a_{\nu l m}-\bar{a}_{\nu}\right|^{2}}{\sigma_{\nu}^{2}},
$$

where $\bar{a}_{\nu}$ is the mean of $a_{\nu l m}$ 's and $\sigma_{\nu}^{2}$ is the variance. If $a_{\nu l m}$ 's are Gaussian then $b_{\nu l m}$ 's obey a $\chi^{2}$ distribution $P(x)=(1 / 2)^{1 / 2} \Gamma(1 / 2) x^{-1 / 2} e^{-x / 2}$ with 1 degree of freedom. To test the goodness of fit between the the theoretical cumulative distribution $I(x)$ and the empirical cumulative distribution function $I_{N}(x)$, we use the Kolmogorov-Smirnov statistic $D_{N}$ which is the least upper bound of all pointwise differences $\left|I_{N}(x)-I(x)\right|[21]$,

$$
D_{N} \equiv \sup _{x}\left|I_{N}(x)-I(x)\right| .
$$

$I_{N}(x)$ is defined as

$$
I_{N}(x)=\left\{\begin{array}{l}
0, \quad x<y_{1}, \\
j / N, \quad y_{j} \leqslant x<y_{j+1}, \quad j=1,2, \ldots, N-1, \\
1, \quad y_{N} \leqslant x,
\end{array}\right.
$$

where $y_{1}<y_{2}<\cdots<y_{N}$ are the computed values of a random sample which consists of $N$ elements. For random variables $D_{N}$ for any $z>0$, it can be shown that the probability of $D_{N}<d$ is given by [22]

$$
\lim _{N \rightarrow \infty} P\left(D_{N}<d=z N^{-1 / 2}\right)=L(z)
$$

where

$$
L(z)=1-2 \sum_{j=1}^{\infty}(-1)^{j-1} e^{-2 j^{2} z^{2}}
$$

From the observed maximum difference $D_{N}=d$, we obtain the significance level $\alpha_{D}=1-P$ which is equal to the probability of $D_{N}>d$. If $\alpha_{D}$ is found to be large enough, the hypothesis $I_{N}(x) \neq I(x)$ is not verified. The significance levels $\alpha_{N}$ for $0 \leqslant l \leqslant 20$ for eigenmodes $k<13$ on the Thurston manifold are shown in Table I. The agreement with the RMT prediction is fairly good for most of eigenmodes which is consistent with the previous computation in Ref. [13]. However, for five degenerated modes, the non-Gaussian signatures are prominent [in Ref. [13], two modes in $(k<10)$ have been missed]. Where does this non-Gaussianity come from?

First of all, we must pay attention to the fact that the expansion coefficients $a_{\nu l m}$ depend on the observing point. In mathematical literature the point is called the base point. For a given base point, it is possible to construct a particular class of fundamental domain called the Dirichlet (fundamental) domain which is a convex polyhedron. A Dirichlet domain $\Omega(x)$ centered at a base point $x$ is defined as

$$
\Omega(x)=\bigcap_{g} H(g, x), \quad H(g, x)=\{z \mid d(z, x)<d[g(z), x]\},
$$

where $g$ is an element of a Kleinian group $\Gamma$ [a discrete isometry group of $\operatorname{PSL}(2, \mathrm{C})]$ and $d(z, x)$ is the proper distance between $z$ and $x$.

The shape of the Dirichlet domain depends on the base point but the volume is invariant. Although the base point can be chosen arbitrarily, it is a standard to choose a point $Q$

TABLE I. Eigenvalues $k$ and the corresponding significance levels $\alpha_{D}$ for the test of the hypothesis $I_{N}(x) \neq I(x)$ for the Thurston manifold. The injectivity radius is maximal at the base point.

\begin{tabular}{cccc}
\hline \hline$k$ & $\alpha_{D}$ & $k$ & $\alpha_{D}$ \\
\hline 5.404 & 0.98 & $10.686(\mathrm{~b})$ & $7.9 \times 10^{-4}$ \\
5.783 & 0.68 & 10.737 & 0.96 \\
$6.807(\mathrm{a})$ & 0.52 & 10.830 & 0.67 \\
6.807 (b) & $7.1 \times 10^{-4}$ & $11.103(\mathrm{a})$ & 0.041 \\
6.880 & 1.00 & $11.103(\mathrm{~b})$ & $8.8 \times 10^{-15}$ \\
7.118 & 0.79 & 11.402 & 0.98 \\
$7.686(\mathrm{a})$ & 0.26 & 11.710 & 0.92 \\
$7.686(\mathrm{~b})$ & $2.3 \times 10^{-8}$ & 11.728 & 0.93 \\
8.294 & 0.45 & 11.824 & 0.31 \\
8.591 & 0.91 & $12.012(\mathrm{a})$ & 0.52 \\
8.726 & 1.00 & $12.012(\mathrm{~b})$ & 0.73 \\
9.246 & 0.28 & 12.230 & 0.032 \\
9.262 & 0.85 & 12.500 & 0.27 \\
9.754 & 0.39 & 12.654 & 0.88 \\
9.904 & 0.99 & 12.795 & 0.76 \\
9.984 & 0.20 & 12.806 & 0.42 \\
10.358 & 0.40 & $12.897(\mathrm{a})$ & 0.87 \\
$10.686(\mathrm{a})$ & 0.76 & $12.897(\mathrm{~b})$ & $6.9 \times 10^{-4}$ \\
\hline
\end{tabular}



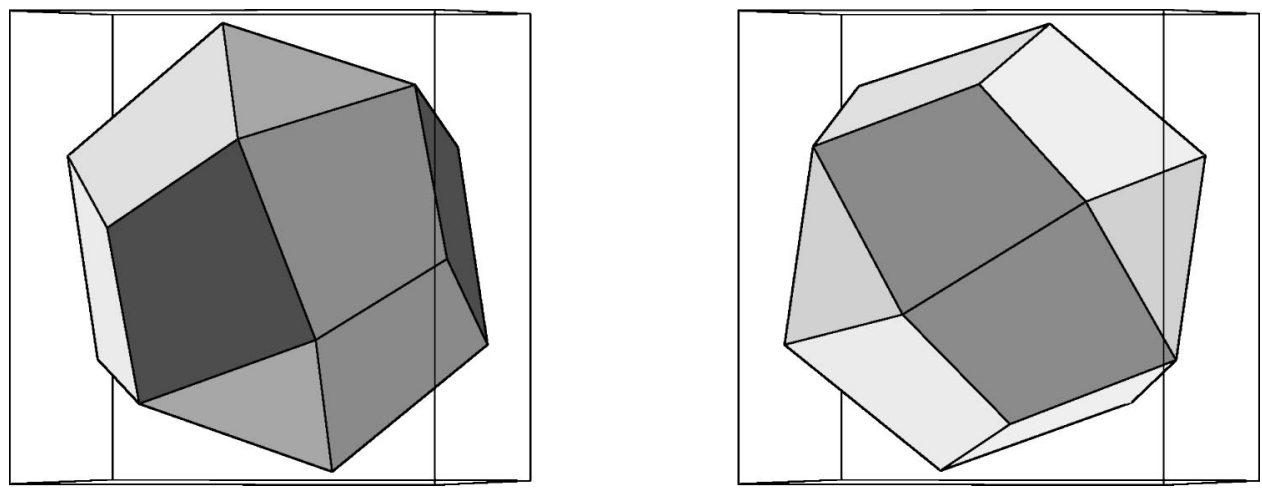

FIG. 4. A Dirichlet domain of the Thurston manifold in the Klein coordinates viewed from opposite directions at $Q$ where the injectivity radius is locally maximal. The Dirichlet domain has a $Z 2$ symmetry (invariant by $\pi$ rotation) at $Q$. where the injective radius ${ }^{1}$ is locally maximal. More intuitively, $Q$ is a center where one can put a largest connected ball on the manifold. If one chooses other point as the base point, the nearest copy of the base point can be much nearer. The reason to choose $Q$ as a base point is that one can expect the corresponding Dirichlet domain to have many symmetries at $Q$ [23].

As shown in Fig. 4, the Dirichlet domain at $Q$ has a $Z 2$ symmetry (invariant by $\pi$ rotation) if all the congruent faces are identified. Generally, congruent faces are distinguished but it is found that these five modes have exactly the same values of eigenmodes on these congruent faces. Then one can no longer consider $a_{\nu l m}$ 's as "independent" random numbers. Choosing the invariant axis by the $\pi$ rotation as the $z$ axis, $a_{\nu l m}$ 's are zero for odd $m$ 's which leads to the observed non-Gaussian behavior. It should be noted that the observed Z2 symmetry is not the subgroup of the isometry group (or symmetry group in mathematical literature) $D 2$ (dihedral group with order 2) of the Thurston manifold since the congruent faces must be actually distinguished in the manifold. ${ }^{2}$

Thus the observed non-Gaussianity is caused by a particular choice of the base point. However, in general, the chance that we actually observe any symmetries (elements of the isometry group of the manifold or the finite sheeted cover of the manifold) is expected to be very low. Because a fixed point by an element of the isometric group is either a part of one-dimensional line (for instance, an axis of a rotation) or an isolated point (for instance, a center of an antipodal map).

In order to confirm that the chance is actually low, the KS statistics $\alpha_{D}$ of $a_{\nu l m}$ 's are computed at 300 base points which are randomly chosen. As shown in Table II, the averaged significance levels $\left\langle\alpha_{D}\right\rangle$ are remarkably consistent with the

\footnotetext{
${ }^{1}$ The injective radius of a point $p$ is equal to half the length of the shortest periodic geodesic at $p$.

${ }^{2}$ The observed Z2 symmetry is considered to be a "hidden symmetry" which is a symmetry of the finite sheeted cover of the manifold (which tessellates the manifold as well as the universal covering space). For instance, the Dirichlet domain of the Thurston manifold can be tessellated by four pieces with three neighboring kitelike quadrilateral faces and one equilateral triangle on the boundary and seven faces which contain the center as a vertex. By identifying the four pieces (by a tetrahedral symmetry), one obtains an orbifold which has a $Z 2$ symmetry.
}

Gaussian prediction. $1 \sigma$ of $\alpha_{D}$ are found to be 0.26 to 0.30 .

Next, we apply the run test for testing the randomness of $a_{\nu l m}$ 's where each set of $a_{\nu l m}$ 's are ordered as $l(l+1)+m$ +1 (see Ref. [21]). Suppose that we have $n$ observations of the random variable $U$ which falls above the median and $\mathrm{n}$ observations of the random variable $L$ which falls below the median. The combination of those variables into $2 n$ observations placed in ascending order of magnitude yields

\section{UUU LL UU LLL UL UU LL.}

Each underlined group which consists of successive values of $U$ or $L$ is called the run. The total number of runs is called the run number. The run test is useful because the run number always obeys the Gaussian statistics in the limit $n \rightarrow \infty$ regardless of the type of the distribution function of the random variables. As shown in Table III, averaged significance levels $\left\langle\alpha_{r}\right\rangle$ are very high ( $1 \sigma$ is 0.25 to 0.31 ). Thus each set of $a_{\nu l m}$ 's ordered as $l(l+1)+m+1$ can be interpreted as a set of Gaussian pseudorandom numbers except for limited choices of the base point where one can observe symmetries of eigenmodes.

Up to now, we have considered $l$ and $m$ as the index numbers of $a_{\nu l m}$ at a fixed base point. However, for a fixed $(l, m)$, the statistical property of a set of $a_{\nu l m}$ 's at a number of different base points is also important since the temperature fluctuations must be averaged all over the places for spatially inhomogeneous models. From Fig. 5, one can see the behavior of $m$-averaged significance levels

$$
\alpha_{D}(\nu, l) \equiv \sum_{m=-l}^{l} \frac{\alpha_{D}\left(a_{\nu l m}\right)}{2 l+1}
$$

which are calculated based on 300 realizations of the base points. It should be noted that each $a_{\nu l m}$ at a particular base point is now considered to be "one realization" whereas a choice of $l$ and $m$ is considered to be "one realization" in the previous analysis (Table I). The agreement with the RMT prediction is considerably good for components $l>1$. For components $l=1$, the disagreement occurs for only several modes. However, the non-Gaussian behavior is distinct in $l$ $=0$ components. What is the reason of the non-Gaussian behavior for $l=0$ ? Let us estimate the values of the expansion coefficients for $l=0$. In general, the complex expansion coefficients $\xi_{\nu l m}$ can be written as 
TABLE II. Eigenvalues $k$ and corresponding averaged significance levels $\left\langle\alpha_{D}\right\rangle$ based on 300 realizations
the base points for the test of the hypothesis $I_{N}(x) \neq I(x)$ for the Weeks and the Thurston manifolds.

\begin{tabular}{|c|c|c|c|c|c|c|c|}
\hline \multicolumn{4}{|c|}{ Weeks } & \multicolumn{4}{|c|}{ Thurston } \\
\hline$k$ & $\left\langle\alpha_{D}\right\rangle$ & $k$ & $\left\langle\alpha_{D}\right\rangle$ & $k$ & $\left\langle\alpha_{D}\right\rangle$ & $k$ & $\left\langle\alpha_{D}\right\rangle$ \\
\hline 5.268 & 0.58 & $10.452(b)$ & 0.62 & 5.404 & 0.63 & $10.686(b)$ & 0.62 \\
\hline 5.737 (a) & 0.61 & 10.804 & 0.63 & 5.783 & 0.61 & 10.737 & 0.62 \\
\hline $5.737(b)$ & 0.61 & 10.857 & 0.62 & 6.807 (a) & 0.62 & 10.830 & 0.63 \\
\hline 6.563 & 0.62 & 11.283 & 0.57 & 6.807 (b) & 0.62 & 11.103 (a) & 0.59 \\
\hline 7.717 & 0.59 & 11.515 & 0.61 & 6.880 & 0.63 & 11.103 (b) & 0.60 \\
\hline 8.162 & 0.61 & 11.726 (a) & 0.63 & 7.118 & 0.61 & 11.402 & 0.61 \\
\hline 8.207 (a) & 0.65 & 11.726 (b) & 0.59 & 7.686 (a) & 0.61 & 11.710 & 0.62 \\
\hline 8.207 (b) & 0.61 & 11.726 (c) & 0.61 & $7.686(b)$ & 0.63 & 11.728 & 0.64 \\
\hline 8.335 (a) & 0.59 & 11.726 (d) & 0.61 & 8.294 & 0.60 & 11.824 & 0.62 \\
\hline 8.335 (b) & 0.62 & 12.031 (a) & 0.60 & 8.591 & 0.60 & 12.012 (a) & 0.63 \\
\hline 9.187 & 0.59 & $12.031(\mathrm{~b})$ & 0.60 & 8.726 & 0.60 & 12.012 (b) & 0.61 \\
\hline 9.514 & 0.56 & 12.222 (a) & 0.61 & 9.246 & 0.60 & 12.230 & 0.60 \\
\hline 9.687 & 0.61 & $12.222(b)$ & 0.62 & 9.262 & 0.63 & 12.500 & 0.63 \\
\hline 9.881 (a) & 0.61 & 12.648 & 0.59 & 9.754 & 0.62 & 12.654 & 0.62 \\
\hline $9.881(\mathrm{~b})$ & 0.62 & 12.789 & 0.59 & 9.904 & 0.60 & 12.795 & 0.62 \\
\hline 10.335 (a) & 0.63 & & & 9.984 & 0.60 & 12.806 & 0.62 \\
\hline $10.335(b)$ & 0.60 & & & 10.358 & 0.62 & 12.897 (a) & 0.62 \\
\hline 10.452 (a) & 0.63 & & & 10.686 (a) & 0.60 & 12.897 (b) & 0.56 \\
\hline
\end{tabular}

For $l=0$, the equation becomes

$$
\xi_{\nu 00}\left(\chi_{0}\right)=-\frac{i}{2 \sqrt{2}} \frac{\sinh \chi_{0}}{\sin \nu \chi_{0}} \int u_{\nu}\left(\chi_{0}, \theta, \phi\right) d \Omega
$$

Taking the limit $\chi_{0} \rightarrow 0$, one obtains
Thus $a_{\nu 00}$ can be written in terms of the value of the eigenmode at the base point. As shown in Fig. 1, the lowest eigenmodes have only one "wave" on scale of the topological identification scale $L$ (which will be defined later on) inside a single Dirichlet domain which implies that the random behavior within the domain may be not present. Therefore, for

TABLE III. Eigenvalues $k$ and corresponding averaged significance levels $\left\langle\alpha_{r}\right\rangle$ for the test of the hypothesis that the $a_{\nu l m}$ 's are not random numbers for the Weeks and Thurston manifolds. $\alpha_{r}$ 's at 300 points which are randomly chosen are used for the computation.

\begin{tabular}{lccccccc}
\hline \hline & \multicolumn{2}{c}{ Weeks } & \multicolumn{5}{c}{ Thurston } \\
\multicolumn{1}{c}{$k$} & $\left\langle\alpha_{r}\right\rangle$ & $k$ & $\left\langle\alpha_{r}\right\rangle$ & $k$ & $\left\langle\alpha_{r}\right\rangle$ & $k$ & $\left\langle\alpha_{r}\right\rangle$ \\
\hline 5.268 & 0.51 & 10.452 (b) & 0.52 & 5.404 & 0.48 & 10.686 (b) & 0.51 \\
5.737 (a) & 0.48 & 10.804 & 0.52 & 5.783 & 0.45 & 10.737 & 0.49 \\
5.737 (b) & 0.45 & 10.857 & 0.53 & 6.807 (a) & 0.53 & 10.830 & 0.53 \\
6.563 & 0.54 & 11.283 & 0.49 & 6.807 (b) & 0.50 & 11.103 (a) & 0.52 \\
7.717 & 0.50 & 11.515 & 0.51 & 6.880 & 0.47 & 11.103 (b) & 0.53 \\
8.162 & 0.54 & 11.726 (a) & 0.51 & 7.118 & 0.50 & 11.402 & 0.51 \\
8.207 (a) & 0.52 & 11.726 (b) & 0.48 & 7.686 (a) & 0.49 & 11.710 & 0.51 \\
8.207 (b) & 0.49 & 11.726 (c) & 0.49 & 7.686 (b) & 0.52 & 11.728 & 0.49 \\
8.335 (a) & 0.53 & 11.726 (d) & 0.48 & 8.294 & 0.50 & 11.824 & 0.54 \\
8.335 (b) & 0.50 & 12.031 (a) & 0.54 & 8.591 & 0.50 & 12.012 (a) & 0.51 \\
9.187 & 0.53 & 12.031 (b) & 0.51 & 8.726 & 0.51 & 12.012 (b) & 0.49 \\
9.514 & 0.55 & 12.222 (a) & 0.54 & 9.246 & 0.43 & 12.230 & 0.51 \\
9.687 & 0.53 & 12.222 (b) & 0.50 & 9.262 & 0.50 & 12.500 & 0.48 \\
9.881 (a) & 0.51 & 12.648 & 0.54 & 9.754 & 0.54 & 12.654 & 0.48 \\
9.881 (b) & 0.51 & 12.789 & 0.48 & 9.904 & 0.52 & 12.795 & 0.50 \\
10.335 (a) & 0.54 & & & 9.984 & 0.49 & 12.806 & 0.51 \\
10.335 (b) & 0.51 & & & 10.358 & 0.53 & 12.897 (a) & 0.57 \\
10.452 (a) & 0.53 & & & 10.686 (a) & 0.51 & 12.897 (b) & 0.55 \\
\hline \hline
\end{tabular}




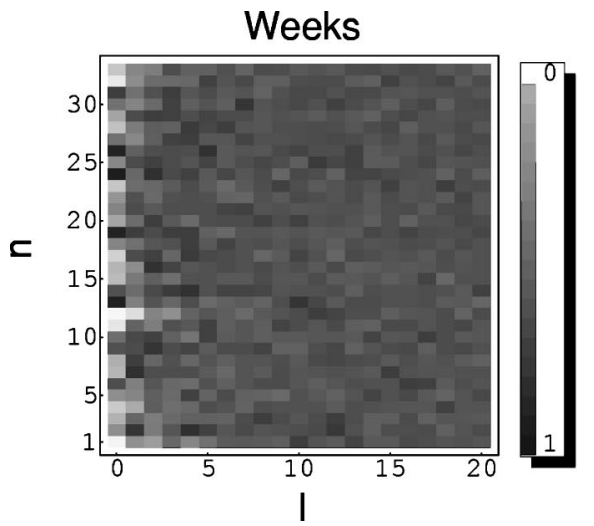

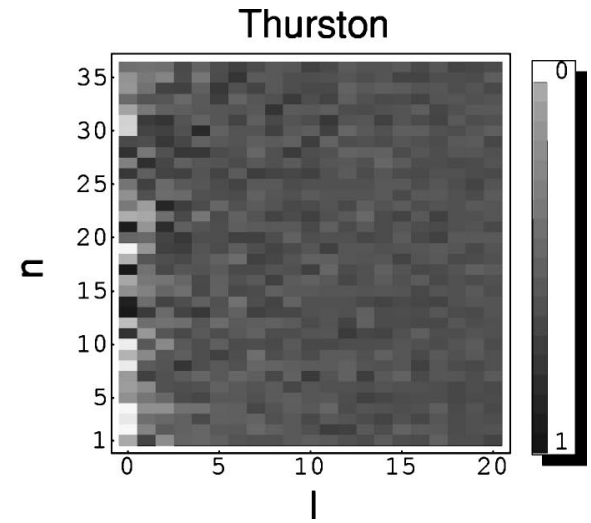

FIG. 5. Plots of $m$ averaged significance levels $\alpha_{D}(\nu, l)$ based on 300 realizations for the Weeks and the Thurston manifolds $(0 \leqslant l$ $\leqslant 20$ and $k<13$ ). $n$ denotes the index number which corresponds to an eigenmode $u_{k}$ where the number of eigenmodes less than $k$ is equal to $n[k(n=1)$ is the lowest nonzero eigenvalue]. The accompanying palettes show the correspondence between the level of the gray and the value. low-lying eigenmodes, one would generally expect nonGaussianity in a set of $a_{\nu 00}$ 's. However, for high-lying eigenmodes, this may not be the case since these modes have a number of "waves" on scale of $L$ and they may change their values locally in an almost random fashion.

The above argument cannot be applicable to $a_{\nu l m}$ 's for $l$ $\neq 0$ where $X_{\nu l}$ approaches zero in the limit $\chi_{0} \rightarrow 0$ while the integral term

$$
\int u_{\nu}\left(\chi_{0}, \theta, \phi\right) Y_{l m}^{*}(\theta, \phi) d \Omega
$$

also goes to zero because of the symmetric property of the spherical harmonics. Therefore $a_{\nu l m}$ 's cannot be written in terms of the local value of the eigenmode for $l \neq 0$. For these modes, it is better to consider the opposite limit $\chi_{0} \rightarrow \infty$. It is numerically found that the sphere with very large radius $\chi_{0}$ intersects each copy of the Dirichlet domain almost randomly (the pulled back surface into a single Dirichlet domain chaotically fills up the domain). Then the values of the eigenmodes on the sphere with very large radius vary in an almost random fashion. For large $\chi_{0}$, we have

$$
X_{\nu l}\left(\chi_{0}\right) \propto e^{-2 \chi_{0}+\phi(\nu, l) i},
$$

where $\phi(\nu, l)$ describes the phase factor. Therefore, the order of the integrand in Eq. (14) is approximately $e^{-2 \chi_{0}}$ since Eq. (14) does not depend on the choice of $\chi_{0}$. As the spherical harmonics do not have correlation with the eigenmode $u_{\nu}\left(\chi_{0}, \theta, \phi\right)$, the integrand varies almost randomly for different choices of $(l, m)$ or base points. Thus we conjecture that Gaussianity of $a_{\nu l m}$ 's have their origins in the chaotic property of the sphere with large radius in $\mathrm{CH}$ spaces. The property may be related to the classical chaos in geodesic flows. ${ }^{3}$

So far we have seen the Gaussian pseudorandomness of the $a_{\nu l m}$ 's. Let us now consider the statistical properties of

\footnotetext{
${ }^{3}$ If one considers a great circle on a sphere with large radius, the length of the circle is very long except for rare cases in which the circle "comes back" before it wraps around in the universal covering space. Because the long geodesics in $\mathrm{CH}$ spaces chaotically (with no particular direction and position) wrap through the manifold, it is natural to assume that the great circles also have this chaotic property.
}

the expansion coefficients. As the eigenmodes have oscillatory features, it is natural to expect that the averages are equal to zero. In fact, the averages of $\left\langle a_{\nu l m}\right\rangle$ 's over $0 \leqslant l$ $\leqslant 20$ and $-l \leqslant m \leqslant-l$ and 300 realizations of base points for each $\nu$ mode are numerically found to be $0.006 \pm 0.04$ $-0.02(1 \sigma)$ for the Weeks manifold, and $0.003 \pm 0.04$ $-0.02(1 \sigma)$ for the Thurston manifold. Let us next consider the $\nu$ dependence ( $k$ dependence) of the variances $\operatorname{Var}\left(a_{\nu l m}\right)$. In order to crudely estimate the $\nu$ dependence, we need the angular size $\delta \theta$ of the characteristic length of the eigenmode $u_{\nu}$ at $\chi_{0}[13]$

$$
\delta \theta^{2} \approx \frac{16 \pi^{2} \operatorname{Vol}(M)}{k^{2}\left\{\sinh \left[2\left(\chi_{0}+r_{\text {ave }}\right)\right]-\sinh \left[2\left(\chi_{0}-r_{\text {ave }}\right)\right]-4 r_{\text {ave }}\right\}},
$$

where $\operatorname{Vol}(M)$ denotes the volume of a manifold $M$ and $r_{\text {ave }}$ is the averaged radius of the Dirichlet domain. There is an arbitrariness in the definition of $r_{\text {ave }}$. Here we define $r_{\text {ave }}$ as the radius of a sphere with volume equivalent to the volume of the manifold

$$
\operatorname{Vol}(M)=\pi\left[\sinh \left(2 r_{\text {ave }}\right)-2 r_{\text {ave }}\right]
$$

which does not depend on the choice of the base point. The topological identification length $L$ is defined as $L=2 r_{\text {ave }}$. For the Weeks and the Thurston manifold, $L=1.19$ and $L$ $=1.20$, respectively. From Eq. (19), for large $\chi_{0}$, one can approximate $u_{\nu}\left(\chi_{0}\right) \sim u_{\nu}^{\prime}\left(\chi_{0}^{\prime}\right)$ by choosing an appropriate radius $\chi_{0}^{\prime}$ which satisfies $\nu^{-2} \exp \left(-2 \chi_{0}\right)=\nu^{\prime-2} \exp \left(-2 \chi_{0}^{\prime}\right)$. Averaging Eq. (14) over $l$ 's and $m$ 's or the base points, for large $\chi_{0}$, one obtains

$$
\left\langle\left|\xi_{\nu^{\prime} l m}\right|^{2}\right\rangle \sim \frac{\exp \left(-2 \chi_{o}\right)}{\exp \left(-2 \chi_{o}^{\prime}\right)}\left\langle\left|\xi_{\nu l m}\right|^{2}\right\rangle
$$

which gives $\left\langle\left|\xi_{\nu l m}\right|^{2}\right\rangle \sim \nu^{-2}$. Thus the variance of $a_{\nu l m}$ 's is proportional to $\nu^{-2}$. The numerical results for the two $\mathrm{CH}$ manifolds shown in Fig. 6 clearly support the $\nu^{-2}$ dependence of the variance.

As we have seen, the property of eigenmodes on general $\mathrm{CH}$ manifolds is summarized in the following conjecture.

Conjecture: Except for the base points which are too close to any fixed points by symmetries, for a fixed $\nu$, a set of the 

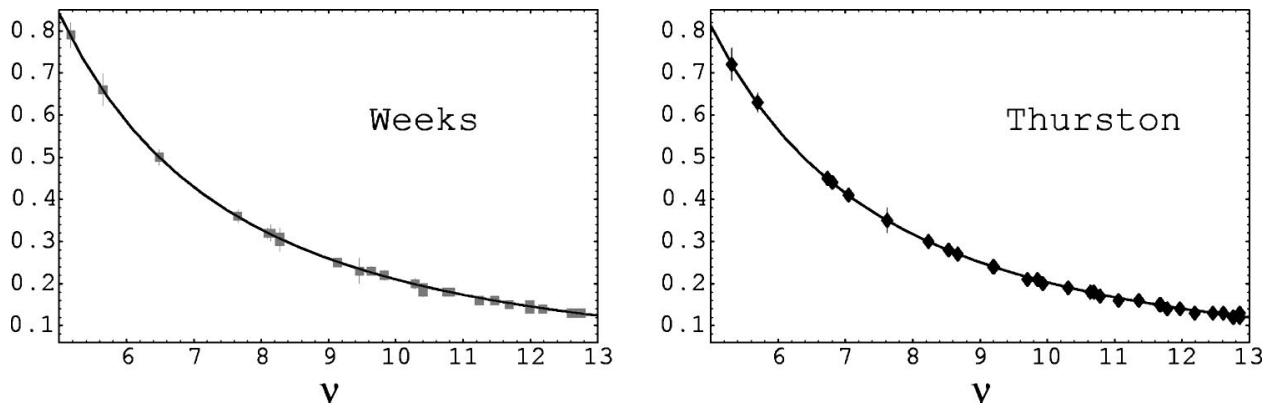

FIG. 6. Averaged squared $a_{\nu}$ 's $(k<13)$ based on 300 realizations of the base points for the Weeks and the Thurston manifold with $\pm 1 \sigma$ run-to-run variations. $a_{\nu}$ is defined to be $\operatorname{Var}\left(a_{\nu l m}\right)$ averaged over $0 \leqslant l \leqslant 20$ and $-l \leqslant m \leqslant l$. The best-fit curves for the Weeks and the Thurston manifolds are $21.0 \nu^{-2}$ and $20.3 \nu^{-2}$, respectively.

expansion coefficients $a_{\nu l m}$ over $(l, m)$ 's can be considered as Gaussian pseudorandom numbers. For a fixed $(\nu l m)(l$ $>0$ ), the expansion coefficients at different base points that are randomly chosen can also be considered as Gaussian pseudorandom numbers. In either case, the variance is proportional to $\nu^{-2}$ and the average is zero.

\section{NON-GAUSSIANITY IN OBSERVABLE ANGULAR POWER SPECTRA}

As mentioned in the last section, perturbations in $\mathrm{CH}$ models are written in terms of linear combinations of eigenmodes and the time evolution of the perturbations. Because the time evolution of the perturbations coincides with that in open models, once the expansion coefficients $\xi_{\nu l m}$ (or $a_{\nu l m}$ ) are given, the evolution of perturbations in $\mathrm{CH}$ models can be readily obtained.

If one assumes that the perturbation is a adiabatic scalar type without anisotropic pressure, and the subhorizon effects such as acoustic oscillations of the temperature and the velocity of the bulk fluid, and the effect of the radiation contribution at high $z$ are negligible, the time evolution of the growing mode of the Newtonian curvature $\Phi$ is analytically given as (see, e.g., Refs. [24,25])

$$
\Phi(\eta)=\frac{5\left(\sinh ^{2} \eta-3 \eta \sinh \eta+4 \cosh \eta-4\right)}{(\cosh \eta-1)^{3}},
$$

where $\eta$ denotes the conformal time. In terms of $\Phi$, the temperature fluctuation in the sky are written as

$$
\frac{\Delta T(\mathbf{n})}{T}=\sum_{l m} a_{l m} Y_{l m}(\mathbf{n})=\sum_{\nu l m} \Phi_{\nu}(0) \xi_{\nu l m} F_{\nu l}\left(\eta_{0}\right) Y_{l m}(\mathbf{n}),
$$

where

$$
\begin{aligned}
F_{\nu l}\left(\eta_{0}\right) \equiv & -\frac{1}{3} \Phi\left(\eta_{*}\right) X_{\nu l}\left(\eta_{0}-\eta_{*}\right) \\
& -2 \int_{\eta_{*}}^{\eta_{0}} d \eta \frac{d \Phi}{d \eta} X_{\nu l}\left(\eta_{0}-\eta\right) .
\end{aligned}
$$

Here $\Phi_{\nu}(0)$ is the initial value of the curvature perturbation and $\eta_{*}$ and $\eta_{0}$ are the conformal time of the last scattering and the present conformal time, respectively. The angular power spectrum $C_{l}$ is defined as

$$
\begin{aligned}
(2 l+1) C_{l} & =\sum_{m=-l}^{l}\left\langle\left|a_{l m}\right|^{2}\right\rangle \\
& =\sum_{\nu, m} \frac{4 \pi^{4} \mathcal{P}_{\Phi}(\nu)}{\nu\left(\nu^{2}+1\right) \operatorname{Vol}(M)}\left\langle\left|\xi_{\nu l m}\right|^{2}\right\rangle\left|F_{\nu l}\left(\eta_{0}\right)\right|^{2},
\end{aligned}
$$

where $\mathcal{P}_{\Phi}(\nu)$ is the initial power spectrum. It should be noted that the above formula converges to that of open models in the short-wavelength limit (summation to integration) provided that $\left\langle\left|\xi_{\nu l m}\right|^{2}\right\rangle$ is proportional to $\nu^{-2}$. The reason is as follows: Let us denote the number of eigenmodes with eigenvalues equal to or less than $\nu$ by $N(\nu)$. In the shortwavelength limit $\nu \gg 1$ one can use Weyl's asymptotic formula which leads to

$$
\frac{d N(\nu)}{d \nu}=\frac{\operatorname{Vol}(M)}{2 \pi^{2}} \nu^{2}
$$

Thus the $\nu^{2}$ dependence in Eq. (26) is exactly cancelled out by the $\nu^{-2}$ dependence of eigenmodes. In what follows we assume the extended Harrison-Zel'dovich spectrum, i.e., $\mathcal{P}_{\Phi}(\nu)=$ const (in the flat limit, it converges to the scale invariant Harrison-Zel'dovich spectrum) as the initial power spectrum.

In estimating the temperature correlations, the nondiagonal terms $\left(l \neq l^{\prime}\right.$ or $\left.m \neq m^{\prime}\right)$ may not be negligible if the background spatial hypersurface is not isotropic, in other words, the angular power spectrum $C_{l}$ may not be sufficient in describing the temperature correlations since $C_{l}$ provides us with only an isotropic information of statistics of the correlations. However, this is not the case for $\mathrm{CH}$ models to which the conjecture proposed in Sec. II is applicable. Based on the Copernican principle, it is not likely that we are at the center of any symmetries. Therefore, in order to statistically estimate the temperature correlations in the globally inhomogeneous background space, one has to consider an ensemble of fluctuations with different initial conditions at different places (or base points) with different orientations. Almost all 

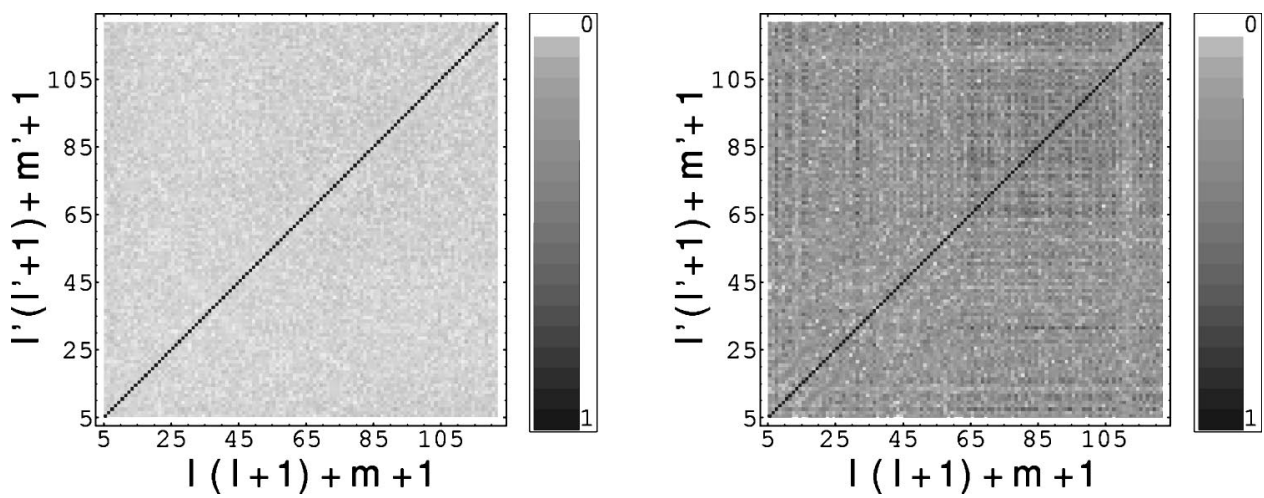

FIG. 7. Contributions of nondiagonal terms in the temperature correlations in unit of diagonal terms which are defined as $f_{l^{\prime} m^{\prime}}^{l m}$ $=\left|\left\langle a_{l m} a_{l^{\prime} m^{\prime}}\right\rangle\right| / \sqrt{\left\langle\left|a_{l m}\right|^{2}\right\rangle\left\langle\left|a_{l^{\prime} m^{\prime}}\right|^{2}\right\rangle}$ for the Thurston model with $\Omega_{0}=0.3$. The four-dimensional space $\left(l, m, l^{\prime}, m^{\prime}\right)$ is represented in the two-dimensional space as $\left(n, n^{\prime}\right)=\left[l(l+1)+m+1, l^{\prime}\left(l^{\prime}+1\right)+m^{\prime}+1\right]$ for $2 \leqslant l \leqslant 10,-l \leqslant m \leqslant l$ and $2 \leqslant l^{\prime} \leqslant 10,-l^{\prime} \leqslant m^{\prime} \leqslant l^{\prime} . f_{l^{\prime} m^{\prime}}^{l m}$ 's are represented by the level of gray shown in the accompanying palettes. The left figure represents $f_{l^{\prime} m^{\prime}}^{l m}$ 's averaged over 300 realizations of the base points with infinite number of initial conditions for the Newtonian curvature. The right figure represents $f_{l^{\prime} m}^{l m}$ ' 's at a base point where the injective radius is maximal with infinite number of initial conditions. The computation is based on 36 eigenmodes $(k<13)$ that are numerically obtained by using the direct boundary element method. The averaged values of the nondiagonal $f_{l^{\prime} m^{\prime}}^{l m}$, s $\left(l \neq l^{\prime}\right.$ or $\left.m \neq m m^{\prime}\right)$ are 0.016 (left) and 0.25 (right).

the anisotropic information is lost in the spatial averaging process since the eigenmodes are Gaussian.

As shown in Fig. 7, for 300 realizations of observing points (left), the averaged absolute values of the off-diagonal elements in unit of diagonal elements are very small $(\sim 0.016)$ whereas their contributions seem to be not negligible $(\sim 0.25)$ at one particular observing point (right) where one can observe a symmetry of the Dirichlet domain. Thus the statistical property of the temperature correlation can be estimated by using $C_{l}$, s provided that the eigenmodes are Gaussian which validates the previous analyses using $C_{l}$ 's for constraining the $\mathrm{CH}$ models [7-10]. The spatial averaging process ${ }^{4}$ must be taken into account since there is no reason to believe that we are in the center of any symmetries.

If the initial conditions satisfy $\left[\Phi_{\nu}(0)\right]^{-2} \propto \nu\left(\nu^{2}+1\right)$ that corresponds to the extended Harrison-Zel'dovich spectrum, then Eq. (23) tells us that the temperature fluctuation is Gaussian since it is equal to a sum of Gaussian (pseudo)random numbers at almost all the observing points. In this case, the Gaussian randomness of the temperature fluctuations in $\mathrm{CH}$ models can be solely attributed to the geometrical property of the space (geometric Gaussianity) which may be related to the deterministic chaos of the corresponding classical system. In other words, the Gaussian randomness can be explained in terms of the classical physical quantities without considering the initial quantum fluctuations provided that the above conditions are initially (deterministically) satisfied.

However, it is natural to assume that $\Phi_{\nu}(0)$ 's are also

\footnotetext{
${ }^{4}$ In general, one should include an averaging process over different choices of orientation of coordinates as well as an averaging process over different choices of the observing point. Nevertheless, the Gaussian conjecture in Sec. II implies that the eigenmodes on $\mathrm{CH}$ spaces are "SO(3) invariant" [12] if averaged all over the space. Therefore, omission of the averaging procedure for different orientations of coordinates make no difference.
}

random Gaussian as in the inflationary scenarios in which Gaussianity (on large scales) of the temperature fluctuations has its origin in Gaussianity of the initial quantum fluctuations because the angular powers are generally similar to the extended Harisson-Zel'dovich spectrum. Then the statistical properties of the temperature fluctuations are determined by the sum of the products of the two independent Gaussian random numbers (the initial fluctuations and the expansion coefficients of the eigenmodes).

Let us calculate the distribution function $F\left(Z, \sigma_{Z}\right)$ of a product of two independent random numbers $X$ and $Y$ that obey the Gaussian (normal) distributions $N\left(X ; 0, \sigma_{X}\right)$ and $N\left(Y ; 0, \sigma_{Y}\right)$, respectively

$$
N(X ; \mu, \sigma) \equiv \frac{1}{\sqrt{2 \pi} \sigma} e^{-(X-\mu)^{2} / 2 \sigma^{2}} .
$$

Then $F\left(Z=X Y, \sigma_{Z}\right)$ is readily given by

$$
\begin{aligned}
F\left(Z, \sigma_{Z}\right) & =2 \int_{0}^{\infty} N\left(Z / Y, 0, \sigma_{X}\right) N\left(Y, 0, \sigma_{Y}\right) \frac{d Y}{Y} \\
& =\frac{1}{\pi \sigma_{X} \sigma_{Y}} K_{0}\left(\frac{|Z|}{\sigma_{X} \sigma_{Y}}\right),
\end{aligned}
$$

where $K_{0}(z)$ is the modified Bessel function. The average of $Z$ is zero and the standard deviation satisfies $\sigma_{Z}=\sigma_{X} \sigma_{Y}$. As is well known, $K_{0}(z)$ is the Green function of the diffusion equation with sources distributed along an infinite line. Although $K_{0}(z)$ diverges at $z=0$ its integration over $(-\infty, \infty)$ is convergent. From the asymptotic expansion of the modified Bessel function 

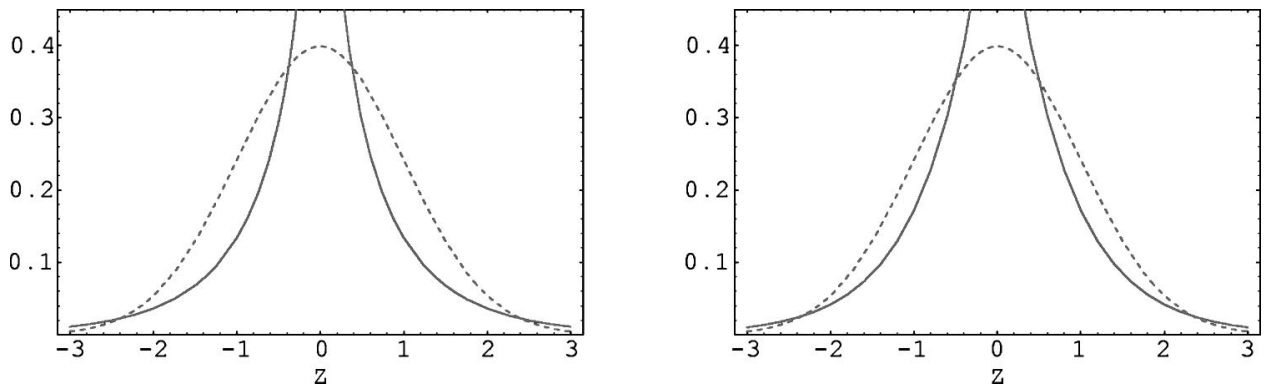

FIG. 8. On the left, the distribution function $F(Z, 1)$ for a product of two random Gaussian numbers is plotted in solid curves. On the right, the distribution function $G(Z, 1)(1 \sigma=1)$ of a sum of two random variables that obey $F(Z, 1 / \sqrt{2})$. The dashed curves represent the Gaussian distribution $N(Z ; 0,1)$.

$$
\begin{aligned}
K_{0}(z) \sim & \sqrt{\frac{\pi}{2 z}} e^{-z}\left[1-\frac{1^{2}}{1 ! 8 z}+\frac{1^{2} \times 3^{2}}{2 !(8 z)^{2}}\right. \\
& \left.-\frac{1^{2} \times 3^{2} \times 5^{2}}{3 !(8 z)^{3}}+\cdots\right], \quad z \gg 1,
\end{aligned}
$$

one obtains in the lowest order approximation

$$
F(Z, \sigma) \sim \frac{1}{\sqrt{2 \pi \sigma|Z|}} e^{-|Z| / \sigma}, \quad Z \gg 1
$$

Thus $F(Z, \sigma)$ is slowly decreased than the Gaussian distribution function with the same variance in the large limit. One can see the two non-Gaussian features in Fig. 8 (left): the divergence at $Z \rightarrow 0$ and the slow convergence to zero at $Z \rightarrow \infty$. The slow convergence is an important feature, as we shall see, in distinguishing the non-Gaussian models with the Gaussian ones. In the modest region $0.4<|Z|<2.4, F(Z, 1)$ is much less than $N(Z, 0,1)$. Generally, the temperature fluctuation is written as a sum of the random variables $Z_{i}$ which obeys the distribution function $F\left(Z_{i}, \sigma_{Z_{i}}\right)$ for a fixed set of cosmological parameters. For large-angle fluctuations, only the eigenmodes with large wavelength $(\equiv 2 \pi / k)$ can contribute to the sum. Due to the finiteness of the space, the number of eigenmodes which dominantly contribute to the sum is finite. Therefore, the fluctuations are distinctively non-Gaussian. For small-angle fluctuations, the number of eigenmodes that contribute to the sum becomes so large that the distribution function converges to the Gaussian distribution as the central limit theorem implies. One can see from Fig. 8 (right) that the distribution function $G(W, 1)$ of $W$ $=Z 1+Z 2$ where both $Z 1$ and $Z 2$ obey $F(Z, \sqrt{2})$ is more similar to the Gaussian distribution $N(Z, 0,1)$ than $F(W, 1)$ in the modest region.

Now let us see the non-Gaussian features of the observable angular power spectrum $\hat{C}_{l}$ assuming that the initial fluctuations are Gaussian. First of all, we define a statistic $\tilde{\chi}^{2} \equiv(2 l+1) \hat{C}_{l} / C_{l}$, where

$$
(2 l+1) \hat{C}_{l}=\sum_{m=-l}^{l} b_{l m}^{2}
$$

If the expansion coefficients $b_{l m}$ of the temperature fluctuation in the sky are Gaussian, $\tilde{\chi}^{2}$ must obey the $\chi^{2}$ distribution with $2 m+1$ degrees of freedom. Figure 9 shows the two non-Gaussian features in the distribution of $b_{l m}$ 's: a slight shift of the peak to the center (zero); slow convergence to zero for large $\tilde{\chi}^{2}$. As shown in Fig. 10, the distribution of $\tilde{\chi}^{2}$ is approximately obtained by assuming that $b_{l m}$ 's obey $G(Z, 1)$ (actually, the distribution functions of $b_{l m}$ 's are slightly much similar to the Gaussian distributions on large angular scales). The two non-Gaussian features are attributed to the nature of the distribution functions of each $b_{l m}$ which give large values at $b_{l m} \sim 0$ and decrease slowly at $b_{l m} \gg 1$ compared with the Gaussian distributions.
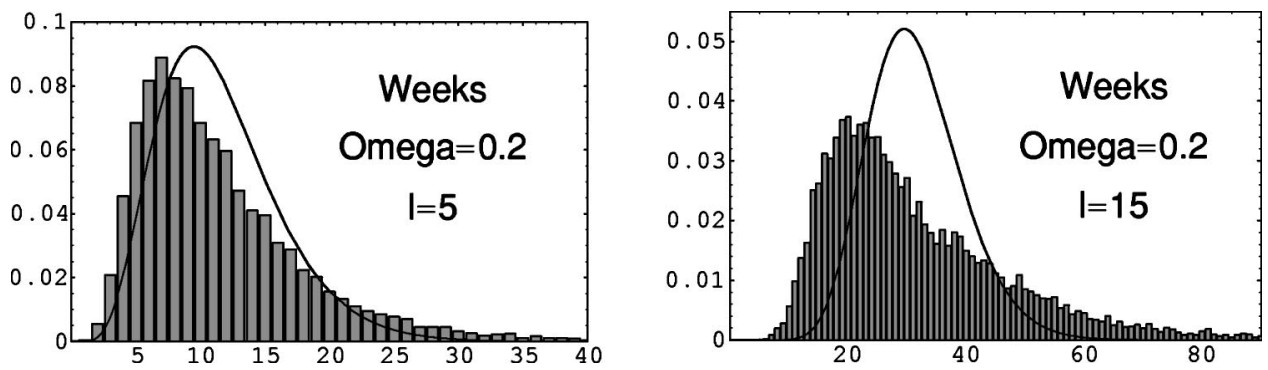

FIG. 9. The distributions of $\tilde{\chi}^{2} \equiv(2 l+1) \hat{C}_{l} / C_{l}$ for the Weeks model with $\Omega_{0}=0.2, l=5$ (left) and 15 (right). The horizontal axes represent the values of $\tilde{\chi}^{2}$. The distributions are calculated using 33 eigenmodes $(k<13)$ based on 200 realizations of the initial Gaussian fluctuations $\Phi_{\nu}(0)$, and 200 realizations of the base points. The contribution of modes $k>13$ is approximately less than $8 \%$ for $l \leqslant 15$. The solid curves represent the $\chi^{2}$ distributions with 11 (left) and 31 (right) degrees of freedom. 

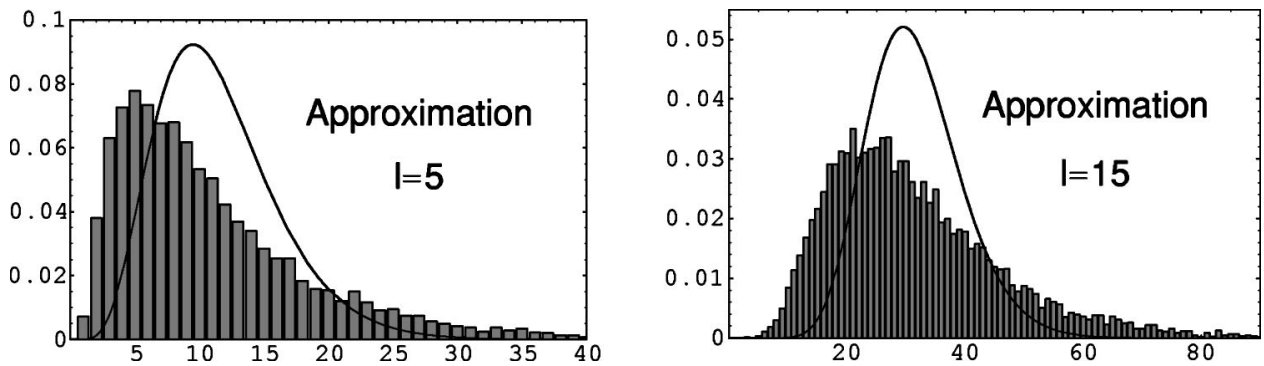

FIG. 10. The distributions of $\tilde{\chi}^{2} \equiv(2 l+1) \hat{C}_{l} / C_{l}$ in an approximated model in which $b_{l m}$ 's obey $G(Z, 1)$ for $l=5$ and $l=15$ based on 40000 realizations for each $b_{l m}$. The horizontal axes represent the values of $\tilde{\chi}^{2}$. The solid curves correspond to the $\chi^{2}$ distributions with 11 (left) and 31 (right) degrees of freedom.

The slow decrease of the distribution of $\tilde{\chi}^{2}$ is important in discriminating the non-Gaussian models with the Gaussian models. As shown in Fig. 11, observing $\tilde{\chi}^{2} \sim 50$ are not improbable for the Weeks $\Omega_{0}$ model $(l=15)$ whereas it is almost unlikely for the Gaussian model. Because the distribution is slowly decreased for large $\tilde{\chi}$, the cosmic variances $\left(\Delta C_{l}\right)^{2}$ are expected to be larger than that of the Gaussian models. From Fig. 12, on large angular scales $(2 \leqslant l \leqslant 15)$, one can see that the standard deviations $\Delta C_{l}$ of $\hat{C}_{l}$ in the two $\mathrm{CH}$ models are approximately 1-2 times of that for the Gaussian models.

\section{TOPOLOGICAL QUANTITIES}

Topological measures:total area of the excursion regions, total length and the genus of the isotemperature contours have been used for testing Gaussianity of the temperature fluctuations in the COBE DMR data [26,27]. Let us first summarize the known results for Gaussian fields (see Refs. $[28,29])$.

The genus $G$ of the excursion set for a random temperature field on a connected and simply connected two-surface can be loosely defined as

$G=$ number of isolated high-temperature connected regions

- number of isolated low-temperature connected regions.

For instance, for a certain threshold, a hot spot will contribute +1 and a cold spot will contribute -1 to the genus. If a

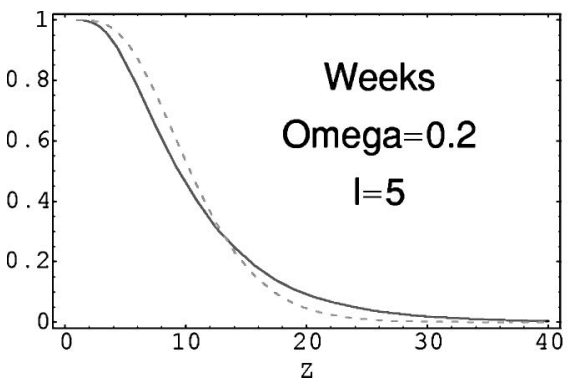

hot spot contains a cold spot, the total contribution to the genus is zero. The genus which is the global property of the random field can be related to the integration of the local properties of the field. From the Gauss-Bonnet theorem, the genus of a closed curve $C$ being the boundary of a simply connected region $\Omega_{C}$ which consists of $N$ arcs with exterior angles $\alpha_{1}, \alpha_{2}, \ldots, \alpha_{N}$ can be written in terms of the geodesic curvature $\kappa_{s}$ and the Gaussian curvature $K$ as

$$
G=\frac{1}{2 \pi}\left[\int_{C} \kappa_{g} d s+\sum_{i=1}^{N} \alpha_{i}+\int_{\Omega_{C}} K d A\right] .
$$

For a random field on the two-dimensional Euclidean space $E^{2}$ where the $\mathrm{N}$ arcs are all geodesic segments (straight line segments), $K$ and $\kappa_{g}$ vanish. Therefore, the genus is written as

$$
G_{E^{2}}=\frac{1}{2 \pi} \sum_{i=1}^{N} \alpha_{i}
$$

The above formula is applicable to the locally flat spaces such as $E^{1} \times S^{1}$ and $T^{2}$ which have $E^{2}$ as the universal covering space since $K$ and $\kappa_{g}$ also vanish in these spaces. In these multiply connected spaces, the naive definition Eq. (32) is not correct for excursion regions surrounded by a loop which cannot be contracted to a point.

In order to compute the genus for a random field on a sphere $S^{2}$ with radius equal to 1 , it is convenient to use a map $\psi: S^{2}-\left\{p_{1}\right\}-\left\{p_{2}\right\} \rightarrow S^{1} \times(0, \pi)$ defined as

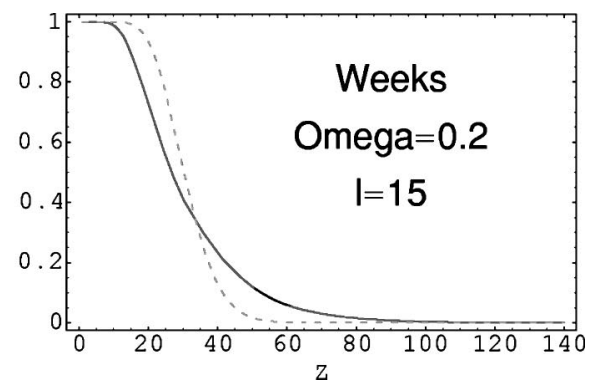

FIG. 11. Plots of $1-P(Z)[P(Z)$ is the cumulative distribution function] which gives the probability of observing $X \geqslant Z$. The solid curves correspond to $1-P\left(\tilde{\chi}^{2}\right)$ for the Weeks model $\Omega_{0}=0.2, l=5$ (left) and $l=15$ (right). The dashed curves correspond to $1-P\left(\chi^{2}\right)$ of the Gaussian model. 

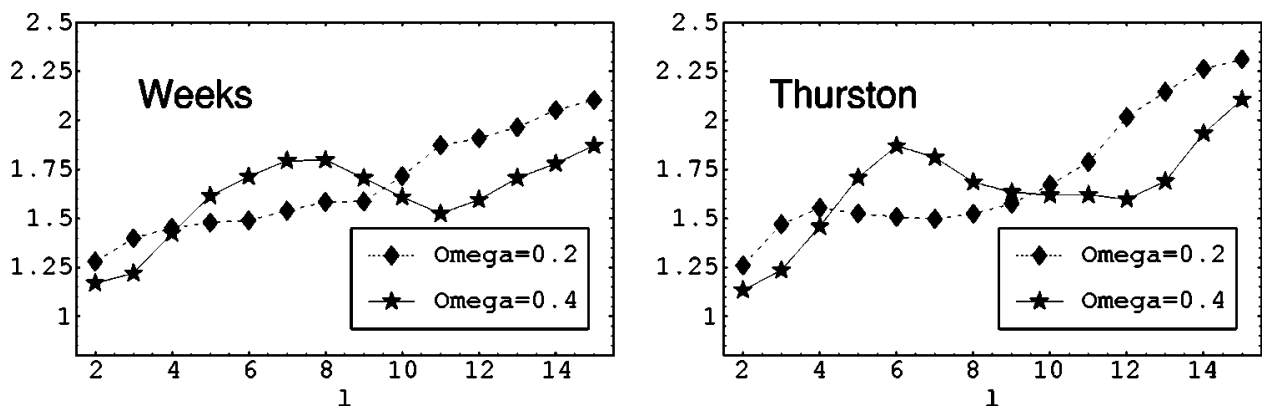

FIG. 12. Plots of $\Delta C_{l}(\mathrm{CH}) /$ $\Delta C_{l}$ (Gauss) for the two $\mathrm{CH}$ models based on 200 realizations of the initial perturbation $\Phi_{\nu}(0)$ and 200 realizations of the base point. $\Delta C_{l}$ denotes the standard deviation $(1 \sigma)$ of $\hat{C}_{l}$. $\psi:(\sin \theta \cos \phi, \sin \theta \sin \phi, \cos \theta) \rightarrow(\phi, \theta)$,

$$
0 \leqslant \phi<2 \pi, \quad 0<\theta<\pi,
$$

where $p_{1}$ and $p_{2}$ denote the north pole and the south pole, respectively. Because $S^{1} \times(0, \pi)$ can be considered as locally flat spaces $(\phi, \theta)$ with metric $d s^{2}=d \theta^{2}+d \phi^{2}$ which have boundaries $\theta=0, \pi$, the genus for excursion regions that do not contain the poles surrounded by straight segments in the locally flat $(\phi, \theta)$ space is given by Eq. (34). It should be noted that the straight segments do not necessarily correspond to the geodesic segments in $S^{2}$. If a pole is inside an excursion region and the pole temperature is above the threshold then the genus is increased by one. If the pole temperature is below the threshold, it does not need any correction. Thus the genus for the excursions is

$$
G_{S^{2}}=\frac{1}{2 \pi} \sum_{i} \alpha_{i}+N_{p},
$$

where $\alpha_{i}$ is the exterior angles at the intersection of two straight segments in the $(\phi, \theta)$ space and $N_{p}$ is the number of poles above the threshold.

Now consider an isotropic and homogeneous Gaussian random temperature field on a sphere $S^{2}$ with radius 1 . Let $(x, y)$ be the local Cartesian coordinates on $S^{2}$ and let the temperature correlation function be $C(r)$ $=\left\langle(\Delta T / T)_{0}(\Delta T / T)_{r}\right\rangle$ with $r=x^{2}+y^{2}$ and $C_{0}=C(0) \equiv \sigma^{2}$, where $\sigma$ is the standard deviation and $C_{2}=-\left(d^{2} C /\right.$ $\left.d r^{2}\right)_{r=0}$. Then the expectation value of the genus for a threshold $\Delta T / T=\nu \sigma$ is given as [29]

$$
\left\langle G_{S^{2}}\right\rangle=\sqrt{\frac{2}{\pi}} \frac{C_{2}}{C_{0}} \nu e^{-\nu^{2} / 2}+\operatorname{erfc}\left(\frac{\nu}{\sqrt{2}}\right),
$$

where $\operatorname{erfc}(x)$ is the complementary error function. The first term in Eq. (37) is equal to the averaged contribution for the
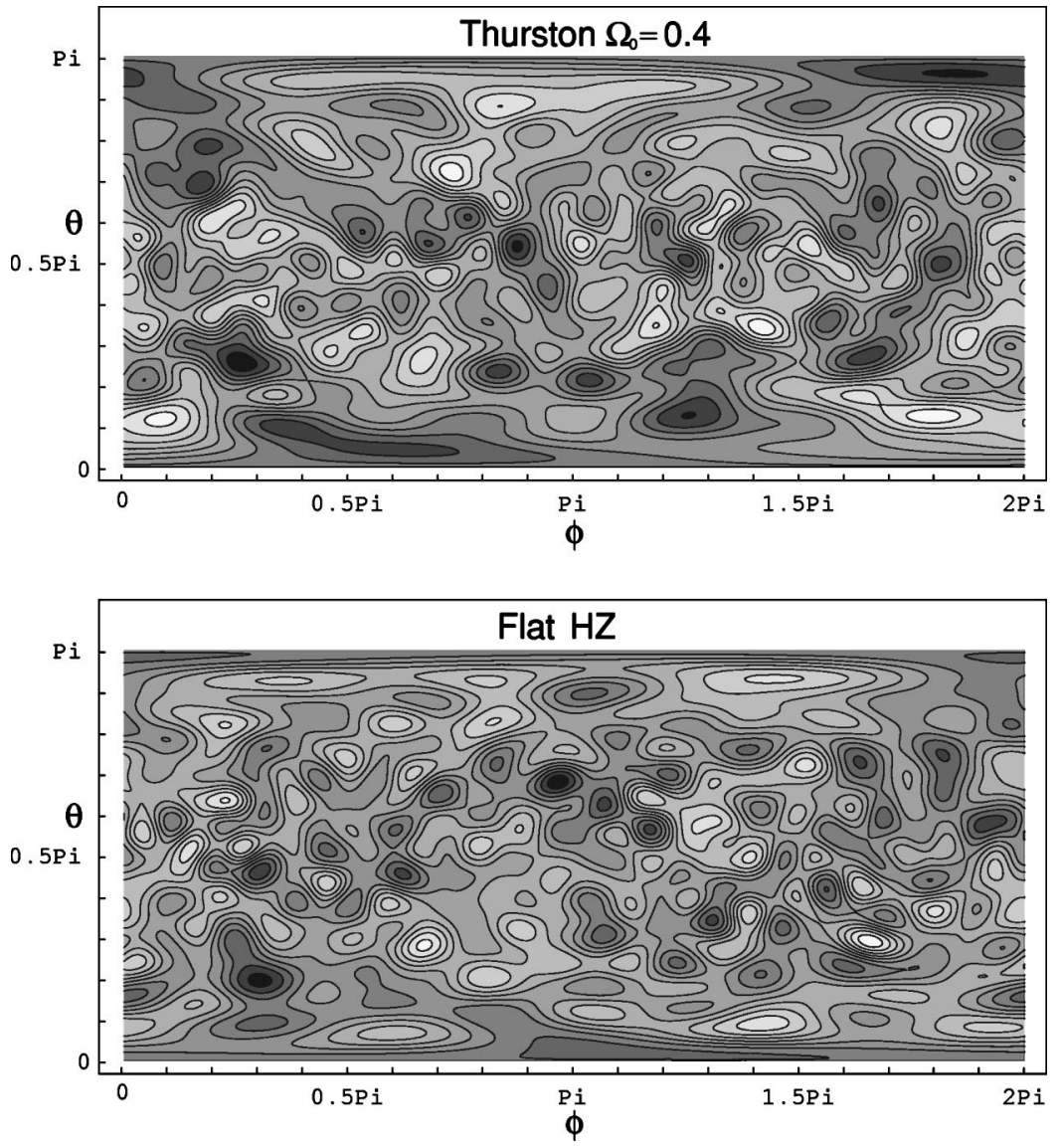

FIG. 13. Contour maps of the CMB (not smoothed by the DMR beam) for the Thurston model $\Omega_{0}=0.4$ and a flat (Einstein-de Sitter) Harrison-Zel'dovich model $C_{l} \propto 1 /[l(l+1)]$ in which all multipoles $l>20$ are removed. 

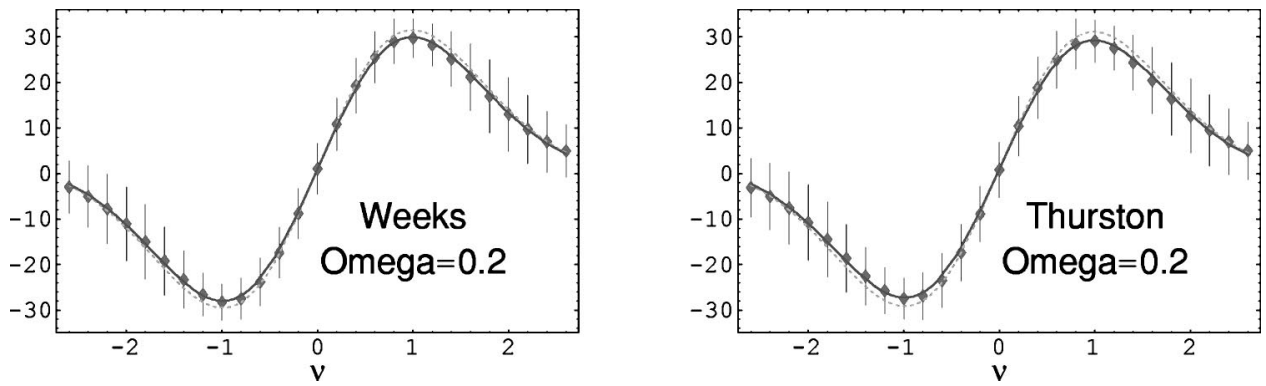

FIG. 14. The mean genuses averaged over 100 realizations of the initial fluctuations and 100 realizations of the base points and $\pm 1 \sigma$ run-to-run variations at 27 threshold levels for the Weeks and the Thurston models with $\Omega_{0}=0.2$. The dashed curves denote the mean values for a Gaussian model where $C_{0}$ and $C_{2}$ are obtained by assuming that the expansion coefficients of the eigenmodes are random Gaussian numbers (the mean is zero and the variance is proportional to $\nu^{-2}$ ). The solid curves denote the mean values for a Gaussian model that are best-fitted to that for $\mathrm{CH}$ models.

excursions which do not contain the poles while the second term in Eq. (37) is the expectation value of $N_{p}$.

The mean contour length per unit area for an isotropic homogeneous Gaussian random field is [28,29]

$$
\langle s\rangle=\frac{1}{2}\left(\frac{C_{2}}{C_{0}}\right)^{1 / 2} e^{-\nu^{2} / 2},
$$

and the mean fractional area of excursion regions for the field is the cumulative probability of a threshold level

$$
\langle a\rangle=\frac{1}{2} \operatorname{erfc}\left(\frac{\nu}{\sqrt{2}}\right),
$$

which gives the second term in Eq. (37).

As in Sec. III, the CMB anisotropy maps for the two $\mathrm{CH}$ adiabatic models are produced by using eigenmodes $k<13$ and angular components $2 \leqslant l \leqslant 20$ for $\Omega_{0}=0.2$ and 0.4 . The contribution of higher modes are approximately 7 and $10 \%$ for $\Omega_{0}=0.2$ and 0.4 , respectively. The initial power spectrum is assumed to be the extended Harrison-Zel'dovich spectrum. The beam-smoothing effect is not included. For comparison, sky maps for the Einstein-de Sitter model with the Harrison-Zel'dovich spectrum $C_{l} \propto 1 /[l(l+1)]$ are also simulated.

In order to compute the genus and the contour length for each model, $10000 \mathrm{CMB}$ sky maps on a $400 \times 200$ grid in

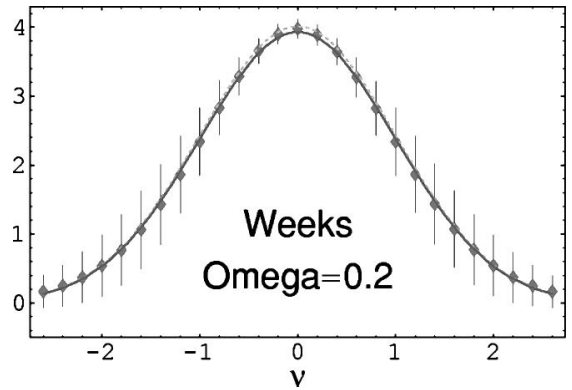

the $(\phi, \theta)$ space are produced. The contours are approximated by oriented straight segments. The genus comes from the sum of the exterior angles at the vertices of the contours and the number of poles at which the temperature is above the threshold. The total contour length is approximated by the sum of all the straight segments. Typical realizations of the sky map are shown in Fig. 13.

Figures 14 and 15 clearly show that the mean genuses and the mean total contours for the two $\mathrm{CH}$ models are well approximated by the theoretical values for the Gaussian models. This is a natural result since the distribution of the expansion coefficients $b_{l m}$ is very similar to the Gaussian distribution in the modest range. On the other hand, at high and low threshold levels, the variances of the total contour lengths and the genuses are larger than that for the Gaussian models that can be attributed to the nature of the distribution function of $b_{l m}$. One can easily see the non-Gaussian signatures from Figs. 16 and 17. The excess variances for the Weeks model $\Omega_{0}=0.4$ compared with the Gaussian flat Harrison-Zel'dovich model are observed at the absolute threshold level approximately $|\nu|>1.4$ for genus and $|\nu|$ $>0.6$ for total contour length. If one assumes that the initial fluctuations are given by $\left[\Phi_{\nu}(0)\right]^{-2} \propto \nu\left(\nu^{2}+1\right)$, the temperature fluctuations for $\mathrm{CH}$ models can be described as Gaussian pseudorandom fields. One can see from Fig. 18 that the behavior of the variances of genus and total contour length for the Gaussian $\mathrm{CH}$ models is very similar to that for

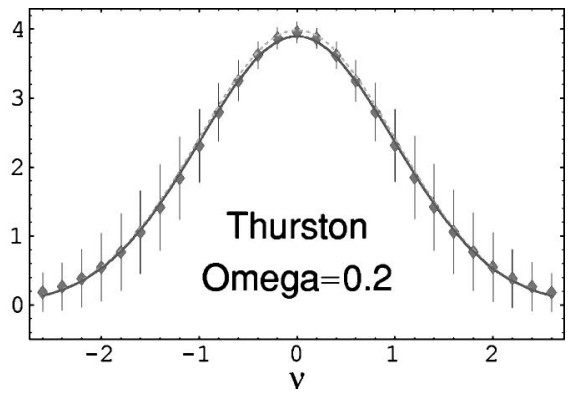

FIG. 15. The mean contour lengths averaged over 100 realizations of the initial fluctuations and 100 realizations of the base points and $\pm 1 \sigma$ run-to-run variations at 27 threshold levels for the Weeks and the Thurston models with $\Omega_{0}=0.2$. The dashed curves denote the mean values for a Gaussian model where $C_{0}$ and $C_{2}$ are obtained by assuming that the expansion coefficients of the eigenmodes are random Gaussian numbers (the mean is zero and the variance is proportional to $\nu^{-2}$ ). The solid curves denote the mean values for a Gaussian model that are best-fitted to that for $\mathrm{CH}$ models. 

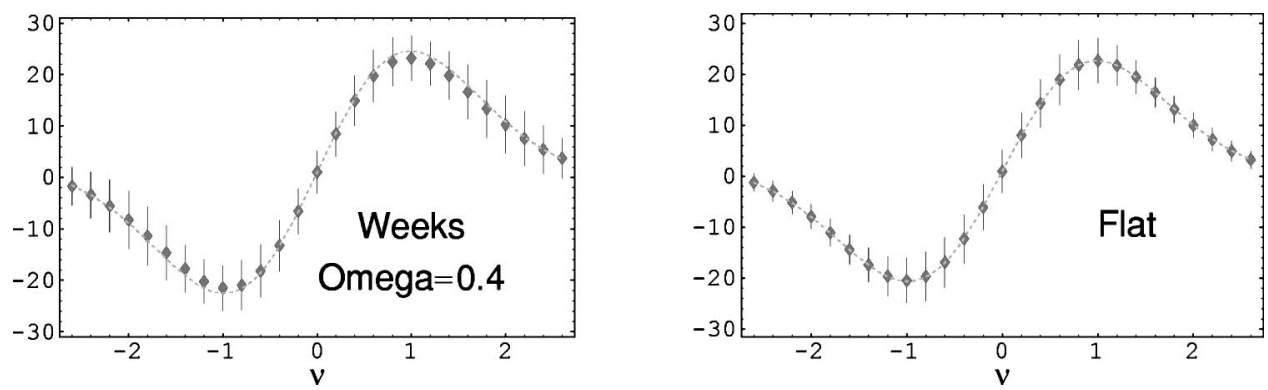

FIG. 16. The mean genuses and $\pm 1 \sigma$ run-to-run variations at 27 threshold levels for a Weeks model with $\Omega_{0}=0.4$ averaged over 100 realizations of the initial fluctuations and 100 realizations of the base points and that for a flat Harisson-Zel'dovich model averaged over 10000 realizations. The dashed curves denote the mean genuses for the corresponding Gaussian models.

the flat Harrison-Zel'dovich model and the variances at high and low threshold levels are considerably smaller than that for the non-Gaussian models.

Because the mean behavior for the two non-Gaussian $\mathrm{CH}$ models is well described by the Gaussian models, the COBE DMR data which exclude grossly non-Gaussian models $[26,27]$ cannot constrain the two $\mathrm{CH}$ models by the topological measurements. However, one should take account of a fact that the signals in the $10^{\circ}$ smoothed COBE DMR fouryear sky maps are comparable to the noises [30] that makes it hard to detect the non-Gaussian signals in the background fluctuations. In fact, some recent works using different statistical tools have shown that the COBE DMR four-year sky maps are non-Gaussian [31-33] although some authors cast doubts upon the cosmological origin of the observed nonGaussian signals $[34,35]$. Thus the evidence of Gaussianity in the CMB fluctuations is still not conclusive.

\section{CONCLUSION}

In this paper, Gaussianity of the eigenmodes and nonGaussianity in the CMB temperature fluctuations in two smallest $\mathrm{CH}$ (Weeks and Thurston) models are investigated. As shown in Sec. II, it is numerically shown that the expansion coefficients of the two $\mathrm{CH}$ spaces behave as if they are random Gaussian numbers at almost all the places. If one recognizes the Laplacian as the Hamiltonian of a free particle, each eigenmode is interpreted as a wave function in a stationary state. The observed behavior is consistent with a prediction of RMT which has been considered to be a good empirical theory to describe the statistical properties of quan-

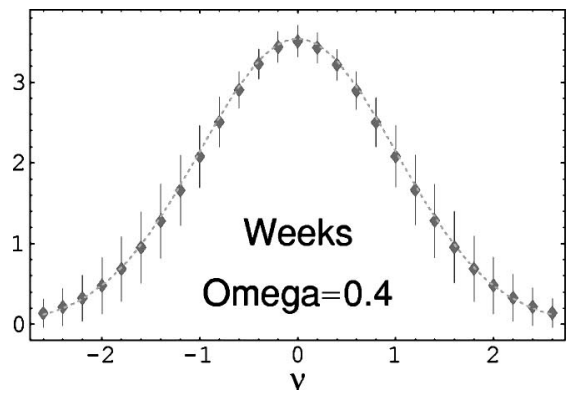

tum mechanical systems whose classical counterparts are strongly chaotic. However, as we have seen, the global symmetries in the system can veil the generic properties. For instance, some eigenmodes on the Thurston manifold have a Z2 symmetry at a point where the injectivity radius is maximal. For these eigenmodes, the expansion coefficients are strongly correlated; hence they can no longer be considered to be random Gaussian numbers.

Because the eigenmodes actually satisfy the periodic boundary conditions, there are points on a sphere $S^{2}$ which are identified with different points on $S^{2}$. These points form pairs of circles which are identified by the periodic boundary conditions [36]. If one could identify all the circles on a sphere, one would be able to construct the corresponding $\mathrm{CH}$ space [37]. Similarly, if one could identify all the fixed points and the corresponding symmetries, one would be able to construct a $\mathrm{CH}$ space which have these symmetries. The observed "randomness" in the eigenmodes is actually determined by these simple structures.

In order to understand the symmetric structures of the $\mathrm{CH}$ spaces, it is useful to choose an observing point (base point) at which one enjoys symmetries as many as possible. However, in reality, there is no natural reason to consider fluctuations at only these particular points since the $\mathrm{CH}$ spaces are globally inhomogeneous.

Since the CMB fluctuations can be written in terms of a linear combination of eigenmodes, the fluctuations in $\mathrm{CH}$ models are almost spatially "isotropic" if averaged all over the space except for very limited places at which the eigenmodes have certain symmetries provided that the eigen-

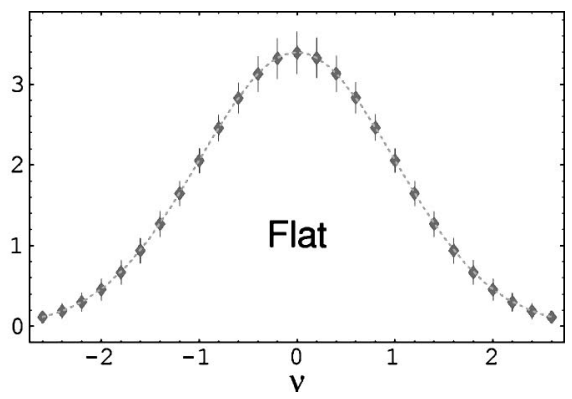

FIG. 17. The mean total contour lengths and $\pm 1 \sigma$ run-to-run variations at 27 threshold levels for a Weeks model with $\Omega_{0}=0.4$ averaged over 100 realizations of the initial fluctuations and 100 realizations of the base points and that for a flat Harisson-Zel'dovich model averaged over 10000 realizations. The dashed curves denote the mean total contour lengths for the corresponding Gaussian models. 

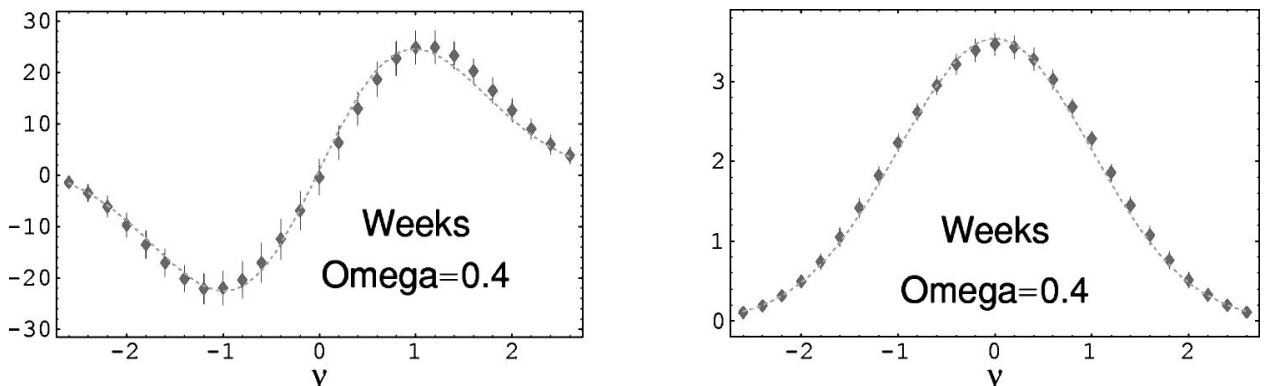

FIG. 18. The mean total contour lengths and genuses and $\pm 1 \sigma$ run-to-run variations at 27 threshold levels for a Weeks model with $\Omega_{0}=0.4$ averaged over 300 realizations of the base points. Here it is assumed that the initial fluctuations deterministically satisfy $\left[\Phi_{\nu}(0)\right]^{-2} \propto \nu\left(\nu^{2}+1\right)$ so that the fluctuations are described by the Gaussian statistics. The dashed curves denote the mean total contour lengths and the mean genuses for the corresponding Gaussian models.

modes are Gaussian. The spatial "isotropy" implies that the contribution of nondiagonal terms in the two-point correlation functions are negligible. Thus the validity of the statistical tests using the angular power spectrum $C_{l}[7-10]$ cannot be questioned on the ground that the background space is anisotropic at a certain point.

If one assumes that the initial fluctuations are Gaussian as in the standard inflationary scenarios, the temperature fluctuations are described by isotropic non-Gaussian random fields since they are written in terms of a sum of products of two independent random Gaussian variables, namely, the initial perturbations and the expansion coefficients of the eigenmodes. The distribution functions of the expansion coefficients $b_{l m}$ for the sky maps at large values are slowly converged to zero than the Gaussian distribution with the same variance and the cosmic variances are found to be larger than that of the Gaussian models.

The increase in the variances are much conspicuous for topological quantities at large or small threshold levels. On the other hand, the mean behavior is well approximated by the Gaussian predictions. Therefore, the obtained results agree with the COBE DMR four-year maps analyzed in Refs. [26,27]. In real observations one has to tackle with what obscure the real signals such as pixel noises, galactic contaminations, beam-smoothing effect and systematic calibration errors which have not been considered in this paper. The absence of large deviations from the mean values at large or small threshold levels in the current data may be due to these effects, which will be much explored in the future work.

Although the recent observations seem to prefer the flat FRW models with the cosmological constant, the evidence is not perfectly conclusive. If one includes the cosmological constant for a fixed curvature radius, the radius of the last scattering surface (horizon) at present in units of curvature radius becomes large. Therefore the observable imprints of the nontrivial topology of the background space become very prominent. For instance, the number $N_{f}$ of copies of the fundamental domains inside the last scattering at the present slice is approximately 27.9 for a Weeks model with $\Omega_{\Lambda}$ $=0.6$ and $\Omega_{m}=0.2$ whereas $N_{f}=4.3$ if $\Omega_{\Lambda}=0$ and $\Omega_{m}$ $=0.8$. Thus we have still great possibilities in detecting the nontrivial topology by the future satellite missions such as the Microwave Anisotropy Probe and Planck which will provide us much better information on the statistical properties of the real signals. The large deviations of the topological quantities from the mean values would be good signals that indicate the hyperbolicity (negative curvature) and the finiteness (smallness) of the universe in addition to the direct observation of the periodic structures peculiar to each nontrivial topology (see Ref. [38] for recent developments).

\section{ACKNOWLEDGMENTS}

I would like to thank Jeff Weeks, Makoto Sakuma, Michihiko Fujii, and Craig Hodgson for answering many questions about symmetric structures of compact hyperbolic threespaces and topology of three-manifolds. I would also like to thank N. J. Cornish, Naoshi Sugiyama, and Kenji Tomita for their informative comments. The numerical computation in this work was carried out at the Data Processing Center in Kyoto University and Yukawa Institute Computer Facility. $\mathrm{K}$. T. Inoue is supported by the JSPS and this work is supported partially by Grant-in-Aid for Scientific Research Fund (Grant No. 9809834).
[1] M. Lachiéze-Rey and J.-P. Luminet, Phys. Rep. 254, 135 (1995).

[2] D. Stevens, D. Scott, and J. Silk, Phys. Rev. Lett. 71, 20 (1993).

[3] A. de Oliveira-Costa, G. F. Smoot, and A. A. Starobinsky, Astrophys. J. 468, 457 (1996).

[4] J. L. Levin, E. Scannapieco, and J. Silk, Phys. Rev. D 58, 103516 (1998).
[5] W. Hu, N. Sugiyama, and J. Silk, Nature (London) 386, 37 (1997).

[6] N. Cornish and D. Spergel, Phys. Rev. D 57, 5982 (1998).

[7] R. Aurich, Astrophys. J. 524, 497 (1999).

[8] K. T. Inoue, in $3 K$ Cosmology, Rome, 1998, edited by L. Maiani, F. Melchiorri, and N. Vittorio, AIP Conf. Proc. No. 476 (AIP, Woodbury, 1999), p. 343.

[9] K. T. Inoue, K. Tomita, and N. Sugiyama, Mon. Not. R. Astron. 
Soc. 314, L21 (2000).

[10] N. J. Cornish and D. N. Spergel, Phys. Rev. D (to be published), astro-ph/9906401.

[11] J. R. Bond, D. Pogosyan, and T. Souradeep, Phys. Rev. D 62, 043006 (2000).

[12] J. C. R. Magueijo, Phys. Lett. B 342, 32 (1995); 352, 499(E) (1995).

[13] K. T. Inoue, Class. Quantum Grav. 16, 3071 (1999).

[14] R. Aurich and F. Steiner, Physica D 64, 185 (1993).

[15] M.L. Mehta, Random Matrices (Academic, New York, 1991).

[16] O. Bohigas, in Proceedings of the 1989 Les Houches School on Chaos and Quantum Physics, edited by A. Giannoni et al. (Elsevier, Amsterdam, 1989).

[17] N. L. Balazs and A. Voros, Phys. Rep. 143, 109 (1986).

[18] T. A. Brody, J. Flores, J. B. French, P. A. Mello, A. Pandy, and S. S. M. Wong, Rev. Mod. Phys. 53, 385 (1981).

[19] J. R. Weeks, SNAPPEA, A Computer Program for Creating and Studying Hyperbolic 3-manifolds, available at URL http:// www.northnet.org/weeks

[20] N. J. Cornish and D. N. Spergel, Report No. math.DG/ 9906017.

[21] R. V. Hogg and E. A. Tanis, Probability and Statistical Inference (Macmillan, New York, 1977).

[22] Z. W. Birnbaum, Introduction to Probability and Mathematical Statistics (Harper Brothers, New York, 1962).

[23] J. R. Weeks (private communication).
[24] H. Kodama and M. Sasaki, Prog. Theor. Phys. Suppl. 78, 1 (1986).

[25] V. F. Mukhanov, H. A. Feldman, and R. H. Brandenberger, Phys. Rep. 215, 203 (1992).

[26] W. N. Colley, J. R. Gott III, and C. Park, Mon. Not. R. Astron. Soc. 281, L82 (1996).

[27] A. Kogut, A. J. Banday, C. L. Bennett, K. M. Górski, G. Hinshaw, G. F. Smoot, and E. L. Wright, Astrophys. J. Lett. 464, L29 (1996).

[28] J. R. Gott III and C. Park, Astrophys. J. 352, 1 (1990).

[29] R. J. Adler, The Geometry of Random Fields (Wiley, Chichester, 1981).

[30] C. L. Bennett et al., Astrophys. J. Lett. 464, L1 (1996).

[31] P. G. Ferreira, J. Magueijo, and K. M. Górski, Astrophys. J. Lett. 503, L1 (1998).

[32] D. Novikov, H. Feldmann, and S. Shandarin, Int. J. Mod. Phys. D 8, 291 (1999).

[33] J. Pando, D. Valls-Gabaud, and L. Z. Fang, Phys. Rev. Lett. 81, 4568 (1998).

[34] B. Bromley and M. Tegmark, Astrophys. J. Lett. 524, L79 (2000).

[35] A. J. Banday, S. Zaroubi, and K. M. Górski, astro-ph/9908070.

[36] N. J. Cornish, D. Spergel, and G. Starkman, Class. Quantum Grav. 15, 2657 (1998); Proc. Natl. Acad. Sci. USA 95, 82 (1998).

[37] J. R. Weeks, Class. Quantum Grav. 15, 2599 (1998).

[38] J. P. Luminet and B. F. Roukema, in Proceedings of Cosmology School held at Cargese, Corsica, 1998, astro-ph/9901364. 